\title{
MASSEY PRODUCTS IN THE COHOMOLOGY OF GROUPS WITH APPLICATIONS TO LINK THEORY
}

\author{
DAVID STEIN
}

\begin{abstract}
Invariants of links in $S^{3}$ are developed using a modification of the Massey product of one-dimensional classes in the cohomology of certain groups. The theory yields two types of invariants, invariants which depend upon a collection of meridians, or basing, of a link, and invariants which do not. The invariants, which are independent of the basing, are compared with John Milnor's $\bar{\mu}$-invariants. For two component links, a collection of ostensibly based invariants is shown to be independent of the basing. If the linking number of the components of such a link is zero, the resulting invariants may be equivalent to the Sato-Levine-Cochran invariants.
\end{abstract}

\section{INTRODUCTION}

Invariants of links in $S^{3}$ are developed using a variation of the Massey product of one-dimensional classes in the cohomology of groups. A basing of a link is a set consisting of exactly one meridian of each component of the link. We obtain a collection of invariants, which depend upon a basing of the link, and a refinement of these which do not. The link invariants are compared with Milnor's $\bar{\mu}$-invariants [8,9]. Porter [10] and Turaev [16] were the first to prove Stallings' conjecture [13] linking the $\bar{\mu}$-invariants and Massey products. For two component links, an infinite family of the based invariants is independent of the basing. These invariants, for two component links such that the linking number of the components is zero, may be equivalent to the Sato-Levine-Cochran invariants $[1,2,12]$.

The present point of view has other implications. It has led to an algebraic means of calculating the invariants and a computer program which does so [15]. Invariants, with no indeterminacy, can be defined for disk links, and the indeterminacy of the invariants for links can be studied as one closes disk links and forms links. An "infinite" invariant may exist when certain of the finite invariants are zero. The effect, on the invariants, of certain alterations of the link may be tractable.

The paper contains four sections. In $\S 1$, a variant of the Massey product of one-dimensional classes in the cohomology of certain groups is shown to contain exactly one element. In $\S 2$, the theory of $\S 1$ is applied to link groups.

Received by the editors July 13, 1988.

1980 Mathematics Subject Classification (1985 Revision). Primary 57M25, 55S30. 
The resulting invariants of links are compared with Milnor's invariants in $\S 3$. In $\S 4$, an infinite family of the based invariants of two component links is shown to be independent of the basing.

Most of this work is part of the author's Ph.D. thesis. He thanks his advisor, Professor Jerome Levine, for his guidance.

\section{The Massey Product INVARIants}

The invariants are defined using a modification of the Massey product.

Definition 1.1 $[5,7]$. Let $(\mathscr{A}, \delta)$ be a differential graded algebra, $q>1$, and $c_{1}, \ldots, c_{q} \in H^{1}(\mathscr{A})$. A defining system, for the Massey product of $\left(c_{1}, \ldots, c_{q}\right)$, is an equivalence class of upper triangular $(q+1) \times(q+1)$ matrices, $\mathbf{M}=\left(m_{i j}\right)$, such that

1. $m_{i i}=1, m_{i j} \in \mathscr{A}^{1}$, and $m_{i i+1}$ represents $c_{i}$;

2. $\delta m_{i j}=\sum_{i<k<j} m_{i k} m_{k j}$ for all $j \neq i+1$, and $(i, j) \neq(1, q+1)$.

Matrices $\mathbf{M}$ and $\mathbf{M}^{\prime}$, which satisfy conditions 1 and 2 , are equivalent if and only if $m_{i j}=m_{i j}^{\prime}$ for all $(i, j) \neq(1, q+1)$. As $\delta$ is a derivation of degree one, $\sum_{i<k<j} m_{i k} m_{k j}$ is a cocycle. Let [M] be the cohomology class $\left[\sum_{1<k<q+1} m_{1 k} m_{k q+1}\right]$. The Massey product of $\left(c_{1}, \ldots, c_{q}\right)$, denoted by $\left\langle c_{1}, \ldots, c_{q}\right\rangle$, is $\left\{[\mathbf{M}] \mid[\mathbf{M}]\right.$ is a defining system for $\left.\left(c_{1}, \ldots, c_{q}\right)\right\}$. Defining systems for the Massey product of a particular ordered set may not exist, or the Massey product may contain more than one element [5, 7]. In the bar resolution of certain groups, with appropriate coefficients, the defining systems can be restricted so that the Massey products of particular ordered sets contain a unique element. This gives rise to the invariants.

Definition 1.2 [3]. Let $q$ be a positive integer. A coefficient system is a collection of ring homomorphisms, $h_{i k j}: R_{i k} \otimes R_{k j} \rightarrow R_{i j} \quad(1 \leq i<k<j \leq q+1)$, such that the diagrams below commute.

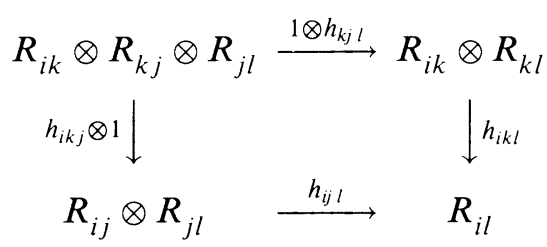

Let $\mathscr{R}$ be a coefficient system. $\mathbf{U}(\mathscr{R}, q+1)$ denotes the group of upper triangular $(q+1) \times(q+1)$ matrices, $\left\{\left(a_{i j}\right) \mid a_{i j} \in R_{i j}\right.$, and $\left.a_{i i}=1\right\}$. The multiplication is matrix multiplication using the $h_{i k j}$. Let $\overline{\mathbf{U}}(\mathscr{R}, q+1)$ denote the group of equivalence classes of elements in $\mathrm{U}$ under the relation $M \equiv M^{\prime}$ if and only if $m_{i j}=m_{i j}^{\prime}$ for all $(i, j) \neq(1, q+1)$. Thus, $\overline{\mathbf{U}}=\mathbf{U} / \operatorname{center}(\mathbf{U})$. One may consider an element of $\overline{\mathbf{U}}$ to be a "matrix" with the upper right-hand entry unspecified.

Let $G$ be a group, $q>1$, and for $1 \leq i \leq q$, let $u_{i} \in H^{1}(G, \mathbf{Z})$. Dwyer [3] shows that defining systems for the Massey product of $\left(u_{1}, \ldots, u_{q}\right)$, 
with coefficients in $\mathbf{Z}$, are in one-to-one correspondence with homomorphisms, $\bar{D}: G \rightarrow \overline{\mathbf{U}}(\mathbf{Z}, q+1)$, such that for each $1 \leq i \leq q$, the homomorphism $\bar{D}_{i i+1}$ is a representative of $-u_{i}$. Let $1 \rightarrow N \rightarrow F \rightarrow G \rightarrow 1$ be a presentation of $G$, and let $h: H_{2}(G, \mathbf{Z}) \rightarrow(N \cap F \sharp F) /(F \sharp N)$ be the Hopf isomorphism. Given $\bar{D}$, there is a homomorphism $D$ such that the diagram below commutes.

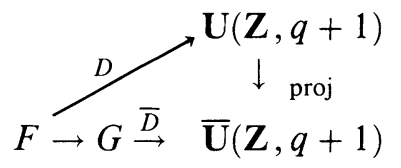

Let $[\bar{D}]$ be the element of $\left\langle u_{1}, \ldots, u_{q}\right\rangle$ obtained from the defining system corresponding to $\bar{D}$, and let $w \in H_{2}(G, \mathbf{Z})$. Dwyer shows that $\langle[\bar{D}], w\rangle=$ $D_{1 q+1}(h(w))$. There is the following naturality result.

Proposition 1.3. Let $f: G \rightarrow H$ be a group homomorphism, $c_{1}, \ldots, c_{q} \in$ $H^{1}(H, \mathbf{Z})$, and $N: H \rightarrow \overline{\mathbf{U}}(\mathbf{Z}, q+1)$ a homomorphism such that $N_{i i+1}$ represents $-c_{i}$. If $M: G \rightarrow \overline{\mathbf{U}}(\mathbf{Z}, q+1)$ is a homomorphism such that $M=N \circ f$, then $[M]=f^{*}[N]$. Note that $M_{i i+1}$ represents $-f^{*}\left(c_{i}\right)$.

We establish the formula for a homomorphism from a free group to $\overline{\mathbf{U}}(\mathbf{Z}, q)$, given the values of the homomorphism on the generators of the group. The formula utilizes the following symbols. $F=\left\langle x_{1}, \ldots, x_{n}\right\rangle$ is the free group on $\left\{x_{1}, \ldots, x_{n}\right\}, \mathbf{Z}\left[\left[t_{1}, \ldots, t_{n}\right]\right]$ is the power series ring in $n$ noncommuting variable with integer coefficients. $\mathscr{M}: F \rightarrow \mathbf{Z}\left[\left[t_{1}, \ldots, t_{n}\right]\right]$ is the Magnus homomorphism defined by $x_{i} \rightarrow 1+t_{i}$.

$$
I(n)=\left\{\left(\tau_{1}, \ldots, \tau_{m}\right) \mid \forall 1 \leq l \leq m, r_{l} \in \mathbf{Z} \text { and } 1 \leq r_{l} \leq n\right\},
$$

and $I(n)_{s}=\left\{\left(r_{1}, \ldots, r_{s}\right) \mid\left(r_{1}, \ldots, r_{s}\right) \in I(n)\right\}$. For $I=\left(r_{1}, \ldots, r_{m}\right) \in I(n)$, let $t_{I}=t_{r_{1}} t_{r_{2}} \cdots t_{r_{m}} \in \mathbf{Z}\left[\left[t_{1}, \ldots, t_{n}\right]\right]$. If $I \neq \varnothing$, and if $\omega \in F, m(I: \omega)$ is the coefficient of $t_{I}$ in $\mathscr{M}(\omega)$, and if $I=\varnothing, m(I: \omega)=1 . S_{i j}=\left\{\left(c_{1}, \ldots, c_{s}\right) \mid \forall l, c_{l}\right.$ is a positive integer, and $\left.\sum_{1}^{s} c_{l}=j-i\right\}$. For $i \leq p \leq j, S_{i j}^{p}=S_{i p} \times S_{p j}$. If, for each $1 \leq i \leq s, f_{i}:\left\{x_{1}, \ldots, x_{n}\right\} \rightarrow \mathbf{Z}$ is a function, and if $I=\left(r_{1}, \ldots, r_{s}\right) \in I(n)_{s}$,

$$
\left(f_{1}, \ldots, f_{s}\right)(I)=f_{1}\left(x_{r_{1}}\right) f_{2}\left(x_{r_{2}}\right) \cdots f_{s}\left(x_{r_{s}}\right) .
$$

Lemma $1.4[10] . \quad m(I: \nu \omega)=\sum_{\left(I_{1}, I_{2}\right)=I} m\left(I_{1}: \nu\right) m\left(I_{2}: \omega\right)$.

Lemma 1.5. For each $1 \leq i<j \leq q+1$, let $a_{i j}:\left\{x_{1}, \ldots, x_{n}\right\} \rightarrow \mathbf{Z}$ be $a$ function. For each $\omega \in F$, define

$$
a_{i j}(\omega)=\sum_{s=1}^{j-i} \sum_{c_{1}, \ldots, c_{s} \in S_{i j}} \sum_{I \in I(n)_{s}}\left(a_{i i+c_{1}} a_{i+c_{1} i+c_{1}+c_{2}} \cdots a_{j-c_{s} j}\right)(I) m(I: \omega) .
$$

Let $A=\left(a_{i j}\right)$; of course, $a_{i i}(\omega)=1$. Then, $A: F \rightarrow \mathbf{U}(\mathbf{Z}, q+1)$ is a homomorphism. Consequently, if $A: F \rightarrow \mathbf{U}(\mathbf{Z}, q+1)$ is a homomorphism, with coordinate functions $a_{i j}$, then $A(\omega)$ is given by the above formula. 
Proof. It suffices to show that $a_{i j}(\nu \omega)=\sum_{p=i}^{j} a_{i p}(\nu) a_{p j}(\omega)$.

$$
\begin{aligned}
& a_{i j}(\nu \omega)=\sum_{s=1}^{j-i} \sum_{c_{1}, \ldots, c_{s} \in S_{i j}} \sum_{I \in I(n)_{s}}\left(\left(a_{i i+c_{1}} \cdots a_{j-c_{s} j}\right)(I)\right. \\
& \left.\times\left(\sum_{\left(I_{1}, I_{2}\right)=I} m\left(I_{1}: \nu\right) m\left(I_{2}: \omega\right)\right)\right) \\
& \sum_{p=i}^{j} a_{i p}(\nu) a_{p j}(\omega) \\
& =\sum_{p=i}^{j}\left[\left(\sum_{s_{1}=1}^{p-i} \sum_{c_{1} \cdots c_{s_{1}} \in S_{i p}} \sum_{I \in I(n)_{s_{1}}}\left(a_{i i+c_{1}} \cdots a_{p-c_{s_{1}} p}\right)(I) m(I: \nu)\right)\right. \\
& \left.\times\left(\sum_{s_{2}=1}^{j-p} \sum_{d_{1}, \ldots, d_{s_{2}} \in S_{p j}} \sum_{J \in I(n)_{s_{2}}}\left(a_{p p+d_{1}} \cdots a_{j-d_{s_{2}} j}\right)(J) m(J: \omega)\right)\right] \\
& =\sum_{p=i}^{j} \sum_{s=1}^{j-i} \sum_{c_{1}, \ldots, c_{s} \in S_{i j}^{p}} \sum_{I \in I(n)_{s}}\left(\left(a_{i i+c_{1}} \cdots a_{j-c_{s} j}\right)(I)\right. \\
& \left.\times m\left(r_{1}, \ldots, r_{l}: \nu\right) m\left(r_{l+1}, \ldots, r_{s}: \omega\right)\right) .
\end{aligned}
$$

Here $I=\left(r_{1}, \ldots, r_{s}\right)$, and $p=c_{1}+\cdots+c_{l}$. The sequence $\left(c_{1}, \ldots, c_{s}\right) \in S_{i j}$ is in $S_{i j}^{p}$ for $p=i+c_{1}, i+c_{1}+c_{2}, \ldots, i+c_{1}+\cdots+c_{s}$. Thus

$$
\begin{aligned}
\sum_{p=i}^{j} a_{i p}(\nu) a_{p j}(\omega) & =\sum_{s=1}^{j-i} \sum_{c_{1}, \ldots, c_{s} \in S_{i j}} \sum_{I \in I(n)_{s}}\left[\left(a_{i+c_{1}} \cdots a_{j-c_{s} j}\right)(I)\right. \\
& \left.\times\left(\sum_{\left(I_{1}, I_{2}\right)=I} m\left(I_{1}: \nu\right) m\left(I_{2}: \omega\right)\right)\right] \\
& =a_{i j}(\nu \omega) .
\end{aligned}
$$

The Massey product invariants of a group are defined relative to a subset.

Definition 1.6. Let $G$ be a group, $\bar{D}: G \rightarrow \overline{\mathbf{U}}(\mathbf{Z}, q+1)$ a homomorphism, and $V \subset G, \bar{D}$ is zero on $V$ if for each $v \in V$ and each $(i, j)$ such that $j \neq i+1, \bar{D}_{i j}(v)=0$.

Let $d$ be an integer, $C=\left(c_{1}, \ldots, c_{q}\right) \in H^{1}(G, \mathbf{Z} / d) \times \cdots \times H^{1}(G, \mathbf{Z} / d)$, and $V \subset G$. An integer $L_{V}(C)$, a coefficient system $R_{V}(C)$, and a subset $T_{V}(C)$ of $H^{2}(G, \mathbf{Z} / d)$ are defined recursively. For $1 \leq a \leq b \leq q$, let $C_{a}^{b}=\left(c_{a}, \ldots, c_{b}\right)$. If $C=\left(c_{1}, c_{2}\right), L_{V}(C)=d, R_{12}=R_{23}=R_{13}=\mathbf{Z} / d$, and $T_{V}(C)=\left\{c_{1} \cdot c_{2}\right\}$. For $q>2$ define $L_{V}(C)$ to be the $\operatorname{gcd}\left\{\langle k, w\rangle, L_{V^{\prime}}\left(C_{1}^{q-1}\right), L_{V^{\prime}}\left(C_{2}^{q}\right) \mid k \in T_{V}\left(C_{1}^{q-1}\right) \cup T_{V^{\prime}}\left(C_{2}^{q}\right)\right.$ and $\left.w \in H_{2}(G, \mathbf{Z} / d)\right\}$. 
$R_{V}(C)_{i j}=\mathbf{Z} / a_{i j}$ where

1. $a_{1 q+1}=L_{V}(C)$;

2. $a_{i i+1}=d$;

3. $a_{i j}=\operatorname{gcd}\left\{L_{V}\left(C_{i}^{j-1}\right),\langle k, w\rangle \mid k \in T_{V}\left(C_{i}^{j-1}\right)\right.$, and $\left.w \in H^{2}(G, \mathbf{Z} / d)\right\}$ for other $(i, j)$.

Note that if $s \leq i<j \leq t$, then $a_{s t} \mid a_{i j}$. Let $h_{i k j}: R_{i k} \otimes R_{k j} \rightarrow R_{i j}$ be the tensor product of the projection maps. Thus, $R_{V}(C)$ is a coefficient system. Define $T_{V}(C)=\left\{[\mathbf{M}] \in H^{2}\left(G, \mathbf{Z} / L_{V}(C)\right) \mid \mathbf{M}\right.$ is a defining system for the massey product of $\left(c_{1}, \ldots, c_{q}\right)$, with coefficients in $R_{V}(C)$, which is zero on $\left.V\right\}$.

The subsets $T_{V}(C)$ contain exactly one element provided $V$ satisfies a certain condition. The lower central series $G_{1} \supset G_{2} \supset \cdots \supset G_{k} \supset \cdots$ of $G$ is defined by $G=G_{1}$, and $G_{k}=\left[G, G_{k-1}\right]$. If $H$ is a subgroup of $G$, and $d$ is an integer, $G \sharp_{d} H$ is the normal subgroup of $G$ generated by $\left\{\left[g_{1}, h\right] g_{2}^{k} \mid g_{1}, g_{2} \in G, h \in H\right.$, and $\left.d \mid k\right\}$ [13].

Definition 1.7. Let $A=\left\langle x_{1}, \ldots, x_{n}\right\rangle$ be the free group on $n$ generators. A subset $V=\left\{v_{1}, \ldots, v_{n}\right\}$ of $G$ has property $M(d)$ provided there is a presentation $1 \rightarrow N \rightarrow F \rightarrow G \rightarrow 1$ of $G$ such that, $\forall q>1$, there is a commutative diagram of exact sequences:

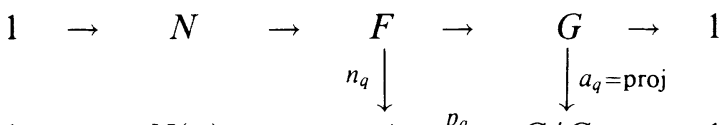

$$
\begin{aligned}
& 1 \rightarrow N(q) \rightarrow A \stackrel{p_{q}}{\rightarrow} G / G_{q} \rightarrow 1
\end{aligned}
$$

and the following propositions are true.

1. $\forall q>1, p_{q}\left(x_{i}\right)=\operatorname{proj}\left(v_{i}\right)$.

2. $\exists\left\{r_{1 q}, \ldots, r_{m q}\right\} \subset A \sharp_{d} A$ such that $N(q)$ is the normal closure of $\left\{r_{1 q}, \ldots, r_{m q}\right\} \cup A_{q}$.

3. $\forall i$ and $q, \exists w_{i q} \in N \cap F \sharp_{d} F$ such that $n_{q}\left(w_{i q}\right) \equiv r_{i q}\left(\bmod A_{q}\right)$.

Lemma 1.8. Suppose $V=\left\{v_{1}, \ldots, v_{n}\right\} \subset G$ satisfies $M(d)$; then

1. $H_{1}(G, \mathbf{Z} / d) \cong(\mathbf{Z} / d)^{n}$.

2. If $D \mid d$, then $H^{2}(G, \mathbf{Z} / D) \cong \operatorname{hom}\left(H_{2}(G, \mathbf{Z} / d), \mathbf{Z} / D\right)$.

3. $H_{2}\left(G / G_{q}, \mathbf{Z} / d\right) \cong\left(N(q) \cap A \sharp_{d} A\right) / A \sharp_{d} N(q)$, and this quotient of subgroups of $A$ is generated by $\left\{r_{i_{q}} \mid 1 \leq i \leq m\right\} \cup A_{q}$.

Proof. Statement 1 follows from condition 2 of the definition, with $q=2$. Statement 2 follows from 1 and the universal coefficient theorem. The isomorphism stated in 3 is the $\bmod d$ Hopf isomorphism. Condition 2 of the definition implies that $N(q)=N(q) \cap A \sharp_{d} A$, and the other half of statement 3 follows.

Theorem 1.9. If $V=\left\{v_{1}, \ldots, v_{n}\right\}$ satisfies $M(d)$, then for all $C=\left(c_{1}, \ldots, c_{q}\right)$ $\in H^{1}(G, \mathbf{Z} / d) \times \cdots \times H^{1}(G, \mathbf{Z} / d)$ :

1. $T_{V}(C)$ contains exactly one element; 
2. $\forall w \in H_{2}(G, \mathbf{Z} / \mathbf{d})$, and $r>1$ :

$$
\left\langle T_{V}(C), w\right\rangle=(-1)^{q} \sum_{I \in I(n)_{q}}\left(a_{q+r}^{*-1}\left(c_{1}\right) \cdots a_{q+r}^{*-1}\left(c_{q}\right)\right)(I) m\left(I: h\left(a_{q+r_{*}}(w)\right)\right) ;
$$

3.

$$
\begin{aligned}
L_{V}(C)=\operatorname{gcd}\left\{d, \sum_{I \in I(n)_{b-a+1}}\left(a_{q+r}^{*-1}\left(c_{a}\right) \cdots a_{q+r}^{*-1}\left(c_{b}\right)\right)(I) m\left(I: r_{j q+r}\right) \mid\right. \\
\\
\quad 1 \leq j \leq m, 1 \leq a<b \leq q+1, \text { and }(a, b) \neq(1, q+1)\} .
\end{aligned}
$$

Proof. For $r \geq 0$ and any coefficient system $\mathscr{R}$ the lower central subgroups, $\overline{\mathbf{U}}(\mathscr{R}, q)_{q+r-1}$, and $\mathbf{U}(\mathscr{R}, q)_{q+r}$ are 1 . Thus a homomorphism $M: G \rightarrow$ $\overline{\mathrm{U}}(\mathscr{R}, q)$ induces a homomorphism $M^{\prime}: G / G_{q+r} \rightarrow \overline{\mathrm{U}}(\mathscr{R}, q)$ and by Proposition 1.3, $a_{q+r}^{*}\left(\left[M^{\prime}\right]\right)=[M]$. Furthermore, $\left[M^{\prime}\right]$ vanishes on $A_{q+r}$, and thus, Lemma 1.8 implies that

$$
\begin{aligned}
\left\langle[M], H_{2}(G, \mathbf{Z} / d)\right\rangle & =\left\langle\left[M^{\prime}\right], a_{q+r_{*}}\left(H_{2}(G, \mathbf{Z} / d)\right)\right\rangle \\
& =\left\langle\left[M^{\prime}\right],\left\{r_{1 q+r}, \ldots, r_{m q+r}\right\}\right\rangle .
\end{aligned}
$$

Lemma 1.8 also implies that

$$
a_{q+r}^{*}: H^{1}\left(G / G_{q+r}, \mathbf{Z} / d\right) \rightarrow H^{1}(G, \mathbf{Z} / d)
$$

is an isomorphism.

The proof is by induction on $q$. Let $q=2$, and let $M$ be a defining system for $\left\langle c_{1}, c_{2}\right\rangle$.

$$
\begin{aligned}
\left\langle T_{V}(C), w\right\rangle & =\langle[M], w\rangle=\left\langle\left[M^{\prime}\right], a_{2+r_{*}}(w)\right\rangle \\
& =\sum_{I \in I(n)_{2}}\left(a_{2+r}^{*-1}\left(c_{1}\right) a_{2+r}^{*-1}\left(c_{2}\right)\right)(I) m\left(I: h\left(a_{2+r_{*}}(w)\right)\right) .
\end{aligned}
$$

The last equality follows from Lemma 1.5.

Let $C=\left(c_{1}, \ldots, c_{q}\right) . \quad L_{V}(C)=\operatorname{gcd}\left\{\langle k, w\rangle \mid k \in T_{V}\left(C_{a}^{b}\right), 1 \leq a<b \leq\right.$ $q,(a, b) \neq(1, q)$, and $\left.w \in H_{2}(G, \mathbf{Z} / d)\right\}$. By induction and the relationships mentioned at the beginning of the proof, statement 3 of the theorem holds.

We show that $T_{V}(C)$ contains at least one element. Let $\mathscr{R}$ denote $R_{V}(C)$. Define $\widetilde{M}: A \rightarrow \overline{\mathbf{U}}(\mathscr{R}, q+1)$ by

$$
\widetilde{M}_{i j}\left(x_{s}\right)=\left\{\begin{array}{l}
-a_{q+r}^{*-1}\left(c_{i}\right)\left(p_{q+r}\left(x_{s}\right)\right), \quad \text { if } j=i+1, \\
0, \quad \text { otherwise. }
\end{array}\right.
$$

Then $\widetilde{M}_{i j}\left(A_{q+r}\right)=0$, and $\widetilde{M}_{i j}\left(r_{l q+r}\right) \equiv 0\left(\bmod a_{i j}\right)$. Thus $\widetilde{M}=M^{\prime} \circ p_{q+r}$. Let $M=M^{\prime} \circ a_{q+r} . M$ is zero on $V$, and thus $[M] \in T_{V}(C) .\langle[M], w\rangle$ is evidently given by statement 2 of the theorem. 
We show that $T_{V}(C)$ contains at most one element. Let $N$ be another defining system for $\left\langle c_{1}, \ldots, c_{q}\right\rangle$ which is zero on $V$. Then $N^{\prime}$ is zero on $\left\{p_{q+r}\left(x_{1}\right), \ldots, p_{q+r}\left(x_{n}\right)\right\}$. Lemma 1.5 implies that $N^{\prime}=M^{\prime}$, and thus, $M=$ $N$.

For the present work, a link is a collection of smoothly embedded, ordered, oriented circles in $S^{3}$.

Proposition 1.10. Let $L \subset S^{3}$ be a link with components $K_{1}, \ldots, K_{n}$. For $1 \leq i \leq n$, let $v_{i}$ be a meridian of $K_{i}$, and let $V=\left\{v_{1}, \ldots, v_{n}\right\} \subset \pi_{1}\left(S^{3}-L, *\right)$.

1. $V$ satisfies property $M(0)$.

2. Let $N$ be the 3-manifold obtained by framed surgery on $L$, and let $d=$ $\operatorname{gcd}\left\{l k\left(K_{i}, K_{j}\right) \mid 1 \leq i \leq j \leq n\right\} . \quad l k\left(K_{i}, K_{j}\right)$ is the linking number of $K_{i}$ and $K_{j}$ if $i \neq j$, while $l k\left(K_{i}, K_{i}\right)$ is the framing number of $K_{i}$. Then $V \subset \pi_{1}(N, *)$ satisfies $M(d)$.

See [9] or $\S 2$ for a proof of 1 , and see $\S 4$ for a proof of 2 .

$T_{V}(C)$ is a based invariant of a link, as it generally depends upon the choice of meridians. One eliminates this dependence by increasing the indeterminacy. Suppose $V=\left\{v_{1}, \ldots, v_{n}\right\} \subset G$ satisfies $M(d)$, then $\left\{v_{1}, \ldots, v_{n}\right\}$ and $\left\{v_{1}^{*}, \ldots, v_{n}^{*}\right\}$ freely generate $H_{1}(G, \mathbf{Z} / d)$ and $H^{1}(G, \mathbf{Z} / d)$, respectively, over $\mathbf{Z} / d$. Let $I=\left(l_{1}, \ldots, l_{q}\right)$ denote $\left(v_{l_{1}}^{*}, \ldots, v_{l_{q}}^{*}\right)$.

Definition 1.11. 1. $\Lambda_{V}(I)=\operatorname{gcd}\left\{\left\langle T_{V}(J), w\right\rangle \mid J\right.$ is a proper subsequence of $I$, and $\left.w \in H_{2}(G, \mathbf{Z} / d)\right\}$.

2. $\sigma_{V}(I)$ is the image of $T_{V}(I)$ in $H^{2}\left(G, \mathbf{Z} / \Lambda_{V}(I)\right)$.

Definition 1.12. Subsets $U=\left\{u_{1}, \ldots, u_{n}\right\}$ and $V=\left\{v_{1}, \ldots, v_{n}\right\}$ of $G$ are conjugate if, $\forall u_{i} \in U, \exists g_{i} \in G$ such that $v_{i}=g_{i} u_{i} g_{i}^{-1}$.

Theorem 1.13. If $U$ and $V$ are conjugate, and $U$ and $V$ satisfy $M(d)$, then for all $I \in I(n), \Lambda_{V}(I)=\Lambda_{U}(I)$, and $\sigma_{V}(I)=\sigma_{U}(I)$.

This theorem follows from two lemmas. For $1 \leq i<j \leq q+1,(i, j) \neq$ $(1, q+1)$, define $b_{i j}=\operatorname{gcd}\left\{\left\langle T_{V}(J), w\right\rangle \mid J\right.$ is a subsequence of $\left(l_{i}, \ldots, l_{j-1}\right)$, and $\left.w \in H^{2}(G, \mathbf{Z} / d)\right\}$, and define $b_{1 q+1}=\operatorname{gcd}\left\{\left\langle T_{V}(J), w\right\rangle \mid J\right.$ is a proper subsequence of $\left(l_{1}, \ldots, l_{q}\right)$, and $\left.w \in H^{2}(G, \mathbf{Z} / d)\right\}$. Note that if $i \leq s<t \leq j$, then $b_{i j} \mid b_{s t}$. Define $R_{i j}=\mathbf{Z} / b_{i j}$, and let $h_{i k j}: R_{i k} \otimes R_{k j} \rightarrow R_{i j}$ be the tensor product of the projection maps. Let $\mathscr{R}=\left(R_{i j}\right)$. Then $\mathscr{R}$ is a coefficient system.

Definition 1.14. Given $I$ as above, a homomorphism $M=\left(m_{i j}\right): G \rightarrow$ $\overline{\mathbf{U}}(\mathscr{R}, q+1)$ is semizero on $V$ for $I$ if

1. $m_{i i+1}$ represents $-v_{l_{i}}^{*}, 1 \leq i \leq q$, and

2. if $p \notin\left\{l_{i}, l_{i+1}, \ldots, l_{j-1}\right\}$, then $\forall i \leq s<t \leq j, m_{s t}\left(v_{p}\right)=0$. 
Lemma 1.15. If $M$ is semizero on $V$ for $I$, then $[M]=\sigma_{V}(I)$.

Proof. Suppose $M$ is semizero on $V$, and $M^{\prime}: G / G_{q+r} \rightarrow \overline{\mathbf{U}}(\mathscr{R}, q+r)$ is the induced homomorphism. Then

$$
\begin{gathered}
\langle[M], w\rangle=\left\langle\left[M^{\prime}\right], a_{q+r_{*}}(w)\right\rangle \\
=\sum_{s=1}^{q} \sum_{c_{1}, \ldots, c_{s} \in S_{1 q+1}} \sum_{J \in I(n)_{s}}\left(m_{11+c_{1}}^{\prime} \cdots m_{q+1-c_{s} q+1}^{\prime}\right)(J) m\left(J: a_{q+r_{*}}(w)\right) . \\
m_{1 k_{1}}^{\prime} \cdots m_{k_{r} k_{q+1}}^{\prime}\left(j_{1}, \ldots, j_{r+1}\right)=0, \text { unless } \\
j_{1} \in\left\{l_{1}, \ldots, l_{k-1}\right\}, \\
j_{2} \in\left\{l_{k}, \ldots, l_{k_{2}-1}\right\}, \\
\vdots \\
j_{r+1} \in\left\{l_{k}, \ldots, l_{q}\right\} .
\end{gathered}
$$

If this is the case, and $J \neq I, m\left(J: a_{q+r_{*}}(w)\right) \equiv 0\left(\bmod b_{1 q+1}\right)$. Thus

$$
\langle[M], w\rangle \equiv m\left(I: a_{q+r_{*}}(w)\right)=\left\langle\sigma_{V}(I), w\right\rangle .
$$

Lemma 1.16. If $U=\left\{u_{1}, \ldots, u_{n}\right\}$ and $V=\left\{v_{1}, \ldots, v_{n}\right\}$ are conjugate subsets of $G$, and $M$ is semizero on $U$ for $I$, then $M$ is semizero on $V$ for $I$.

Proof. Let $M$ be semizero on $V$ for $I$. Since, for each $1 \leq i \leq n, v_{i}$ is a conjugate of $u_{i}, v_{i}^{*}=u_{i}^{*}$. Thus $\left[m_{i i+1}\right]=-u_{l i}^{*}$.

For each $1 \leq i<j \leq q+1,(i, j) \neq(1, q+1)$, deleting rows 1 through $i-1$ and rows $j+1$ through $q+1$, and deleting columns 1 through $i-1$ and columns $j+1$ through $q+1$ defines a homomorphism, $\Phi_{i j}: \overline{\mathbf{U}}(\mathbf{Z}, q+1) \rightarrow \mathbf{U}(\mathbf{Z}, j-i+1)$. Precisely, $\left(\Phi_{i j}(B)\right)_{s t}=b_{s+i-1, i+t-1} . \Phi_{i j}$ is defined similarly for the matrix groups with coefficients in a coefficient system.

Suppose $p \notin\left\{l_{i}, l_{i+1}, \ldots, l_{j-1}\right\}$. Then $\left(\Phi_{i j} \circ M\right)\left(v_{p}\right)=1$. Thus

$$
\begin{aligned}
\left(\Phi_{i j} \circ M\right)\left(u_{p}\right) & =\left(\Phi_{i j} \circ M\right)\left(g_{p} v_{p} g_{p}^{-1}\right) \\
& =\left(\Phi_{i j} \circ M\right)\left(g_{p}\right)\left(\Phi_{i j} \circ M\right)\left(v_{p}\right)\left(\Phi_{i j} \circ M\right)\left(g_{p}^{-1}\right) \\
& =1 .
\end{aligned}
$$

Therefore, $M$ is semizero on $U$ for $I$. Theorem 1.13 follows from these two lemmas.

\section{THE INVARIANTS FOR LINK GROUPS}

In this section Milnor's construction of the $\bar{\mu}$-invariants is outlined, and one sees that any collection of meridians of a link group satisfies $M(0)$. As any two collections of meridians are conjugate, the $\sigma$-invariants, defined above, are independent of the basing. The based invariants are shown to be invariants of based P.L. I-equivalence, while the link invariants are invariants of smooth cobordism and P.L. isotopy. 
J. Milnor defines the $\bar{\mu}$-invariants [8,9]. Let $L$ be an $n$-component link, and for each $1 \leq i \leq n$, let $r_{i}$ be the number of crossings over the $i$ th component in some projection of $L$. Then $G$, the fundamental group of the complement of $L$, has a presentation of the form $\left\langle x_{i j} \mid w_{i j} ; 1 \leq i \leq n, 1 \leq j \leq r_{i}\right\rangle$, where

$$
\begin{aligned}
& w_{i j}=x_{i j+1}^{-1} v_{i j}^{-1} x_{i 1} v_{i j} \quad\left(j \neq r_{i}\right), \\
& w_{i r_{i}}=x_{i 1}^{-1} v_{i r_{i}}^{-1} x_{i 1} v_{i r_{i}} .
\end{aligned}
$$

$\left(x_{i 1}, v_{i r_{i}}\right)$ represents a meridian-longitude pair of the $i$ th component [11]. Let $F$ be the free group $\left\langle x_{i j}\right\rangle$, and let $A$ be the free group $\left\langle x_{1}, \ldots, x_{n}\right\rangle$. For $q \geq 2$, Milnor defines homomorphisms $n_{q}: F \rightarrow A$, so that

1. $n_{q}\left(x_{i 1}\right)=x_{i}$

2. for each $q$, the following diagram commutes:

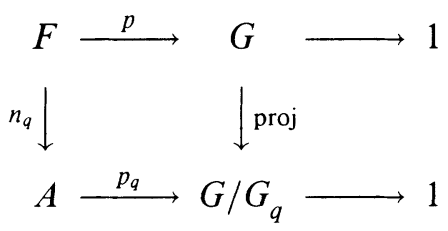

He shows that, for $j \neq r_{i}, n_{q}\left(v_{i j}\right) \equiv 0\left(\bmod A_{q}\right)$, and letting $v_{i q}=n_{q}\left(v_{i r_{i}}\right)$, that $\operatorname{ker}\left(p_{q}\right)$ is the normal subgroup generated by $\left\{\left[x_{i}, v_{i q}\right], A_{q} \mid 1 \leq i \leq n\right\}$. Thus $\left\{p\left(x_{11}\right), \ldots, p\left(x_{n 1}\right)\right\}$, and hence, any collection of meridians satisfies $M(0)$. He also shows that, $\forall r \geq 1, v_{i q} \equiv v_{i q+r}\left(\bmod A_{q}\right)$. Thus, if $s<q$, and $I \in I(n)_{s}, m\left(I: v_{i q}\right)=m\left(I: v_{i q+r}\right)$. He then defines the $\bar{\mu}$-invariants of a link. Let $r \geq 2$, and $I=\left(i_{1}, i_{2}\right) \in I(n) . \bar{\mu}(I)=m\left(i_{1}: v_{i_{2} r}\right)$. Let $q>2, r \geq 0$, and let $I=\left(i_{1}, \ldots, i_{q}\right)$.

$$
\begin{aligned}
& \Delta(I)=\operatorname{gcd}\left\{m\left(s_{a}, \ldots, s_{b}: v_{s_{b+1} q+r}\right) \mid\left(s_{a}, \ldots, s_{b+1}\right)\right. \text { is a cyclic permutation } \\
& \text { of a proper subsequence of } \left.\left(i_{1}, \ldots, i_{q}\right)\right\}, \\
& \bar{\mu}(I)=\text { the residue of } m\left(i_{1}, \ldots, i_{q-1}: v_{i_{q} q+r}\right) \quad(\bmod \Delta(I)) .
\end{aligned}
$$

If $V$ is a collection of meridians of a link group of an $n$-component link, then $V$ satisfies $M(0)$. Thus, for $q>1$ and $C$, a $q$-tuple of cohomology classes, or $I$, an element of $I(n)_{q}$, the invariants $T_{V}(C)$ and $\sigma_{V}(I)$ are defined. As any two collections of meridians are conjugate, one defines $\sigma(I)=\sigma_{V}(I)$.

We show that the invariants are invariants of the equivalence classes of links stated above. Let $N(L)$ be a tubular neighborhood of $L, X=\overline{S^{3}-N(L)}$, and $G=\pi_{1}(X, x)$. The boundary of $X$ is the disjoint union of $n$ torii, $T_{1}, \ldots, T_{n}$, which are ordered and oriented compatibly with the components of $L$. Let $\tau_{j} \in H_{2}(X)$ be the class represented by $T_{j}$. Let $\hbar: H_{2}(X) \rightarrow H_{2}(G)$ be the Hopf map, and let $\omega_{j}=\hbar\left(\tau_{j}\right)$.

Let $L^{0}$ and $L^{1}$ be $n$-component links, and let $V^{0}=\left\{v_{1}^{0}, \ldots, v_{n}^{0}\right\}$ and $V^{1}=\left\{v_{1}^{1}, \ldots, v_{n}^{1}\right\}$ be collections of meridians of $L^{0}$ and $L^{1}$ respectively. 
Definition 2.1. $\left(L^{0}, V^{0}\right)$ and $\left(L^{1}, V^{1}\right)$ are based P.L. $I$-equivalent if

1. there is a proper P.L. embedding $\Phi: \amalg_{j=1}^{n} S^{1} \times I \rightarrow S^{3} \times I$ such that, for $i=0,1, \Phi_{i}$, which is $\Phi \mid \amalg S^{1} \times\{i\}$, is $L^{i}$, and

2. for all $1 \leq j \leq n$ and $q>1, l_{\#}^{0}\left(v_{j}^{0}\right)\left(\bmod G_{q}\right)$. Here $X$ is the complement of a regular neighborhood of the image of $\Phi, X^{i}=X \cap\left(S^{3} \times\{i\}\right), \imath^{i}: X^{i} \rightarrow X$ is inclusion, $G=\pi_{1}(X, x)$, and $G^{i}=\pi_{1}\left(X^{i}, x^{i}\right)$.

Theorem 2.2. 1. If $\left(L^{0}, V^{0}\right)$ and $\left(L^{1}, V^{1}\right)$ are based P.L. I-equivalent, then for all $q$ and all $C=\left(c_{1}, \ldots, c_{q}\right) \in H^{2}(X) \times \cdots \times H^{2}(X), R_{V^{0}}\left(l^{0^{*}}(C)\right)=$ $R_{V^{1}}\left(l^{1^{*}}(C)\right)$, and for all $1 \leq j \leq n$,

$$
\left\langle T_{V^{0}}\left(l^{0^{*}}(C)\right), \omega_{j}^{0}\right\rangle=\left\langle T_{V^{1}}\left(l^{1^{*}}(C)\right), \omega_{j}^{1}\right\rangle .
$$

2. If $L^{0}$ and $L^{1}$ are P.L. isotopic or cobordant, then for all indexing sequences $I$ of $n, \Lambda^{0}(I)=\Lambda^{1}(I)$, and $\left\langle\sigma^{0}(I), \omega_{j}^{0}\right\rangle=\left\langle\sigma^{1}(I), \omega_{j}^{1}\right\rangle$.

Proof of 1 . Note that, for $1 \leq j \leq n, l_{*}^{0}\left(\nu_{j}^{0}\right)=l_{*}^{1}\left(\nu_{j}^{1}\right)$, and $l_{*}^{0}\left(\tau_{j}^{0}\right)=l_{*}^{1}\left(\tau_{j}^{1}\right)$. One inducts on $q$. For $q=2$, the result follows by the naturality of the cup product. By induction, $R_{V^{0}}\left(l^{0^{*}}(C)\right)=R_{V^{1}}\left(l^{1^{*}}(C)\right)$. Denote this coefficient system by $\mathscr{R}$. Stallings' theorem [13] implies that

$$
\overline{l_{\sharp}^{i}}: G^{i} / G_{q}^{i} \rightarrow G / G_{q}
$$

is an isomorphism for all $q>1$. Thus a defining system $\Upsilon^{0}: G^{0} \rightarrow \overline{\mathbf{U}}(\mathscr{R}, q+1)$ for $C^{0}$ induces the commutative diagram:

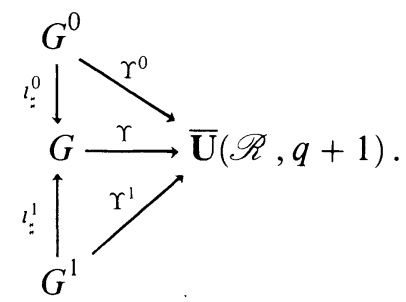

$\Upsilon$ and $\Upsilon^{1}$ are defining systems for $C$ and $C^{1}$ respectively. If $L^{0}$ and $L^{1}$ are based P.L. $I$-equivalent, and $\Upsilon^{0}$ is zero on $V^{0}$, then $\Upsilon$ is zero on $l_{\sharp}^{0}\left(V^{0}\right)$, and $\Upsilon^{1}$ is zero on $V^{1}$. Thus,

$$
\begin{aligned}
\left\langle T_{V^{0}}\left(C^{0}\right), \omega_{j}^{0}\right\rangle & =\left\langle\left[\Upsilon^{0}\right], \omega_{j}^{0}\right\rangle \\
& =\left\langle[\Upsilon], l_{*}^{0}\left(\omega_{j}^{0}\right)\right\rangle \quad \text { (by Proposition 1.3) } \\
& =\left\langle[\Upsilon], l_{*}^{1}\left(\omega_{j}^{1}\right)\right\rangle=\left\langle\left[\Upsilon^{1}\right], \omega_{j}^{1}\right\rangle=\left\langle T_{V^{1}}\left(C^{1}\right), \omega_{j}^{1}\right\rangle,
\end{aligned}
$$

which proves statement 1.

Proof of 2. Suppose that $L^{0}$ and $L^{1}$ are P.L. isotopic or cobordant and $C$ is a collection of the canonical generators of $H^{1}(X)$, then $C^{0}$ and $C^{1}$ are represented by the same sequence $I \in I(n)_{q}$. Furthermore, for each $1 \leq j \leq n$, 
there is an element $g_{j} \in G$ such that $l_{\sharp}^{0}\left(v_{j}^{0}\right)=g_{j} \cdot l_{\sharp}^{1}\left(v_{j}^{1}\right) \cdot g_{j}^{-1}$. Thus, if $\Upsilon^{0}$ is zero on $V^{0}$, then $\Upsilon^{1}$ is semizero on $V^{1}$. Statement 2 follows using the argument above and Lemma 1.16.

\section{COMPARISON OF THE $\bar{\mu}$ AND $\sigma$ INVARIANTS}

Let $L$ be an $n$ component link. In this section the $\bar{\mu}$ and $\sigma$ invariants of $L$ are compared. We prove

Theorem 3.1. If $I=\left(i_{1}, \ldots, i_{q}\right) \in I(n)$, and $i_{1} \neq i_{q}$, then

1. $\Delta(I) \mid \Lambda(I)$,

2. if $i_{\alpha} \neq i_{\beta}$ for all $1 \leq \alpha<\beta \leq q$, then $\Delta(I)=\Lambda(I)$,

3. $\left\langle\sigma(I), \omega_{i_{1}}\right\rangle \equiv-\left\langle\sigma(I), \omega_{i_{q}}\right\rangle(\bmod \Lambda(I))$, and $(-1)^{q}\left\langle\sigma(I), \omega_{i_{q}}\right\rangle \equiv \bar{\mu}(I)$ $(\bmod \Delta(I))$.

Each of the $\bar{\mu}(I)$ compares with a $\sigma\left(I^{\prime}\right)$, where $\bar{I}^{\prime}$ is a cyclic permutation of $I$, since the $\bar{\mu}$-invariants are invariant under cyclic permutation of the indices, and $\bar{\mu}(i, i, \ldots, i)=0$ for all $1 \leq i \leq n$ [9]. The $\sigma$-invariants are not, in general, invariant under cyclic permutation of the indices, and thus, there are links and sequences for which $\Delta$ is a proper divisor of $\Lambda$. We present two links which are distinguished by $\sigma(1,2,3,1,4)$, although they have identical $\bar{\mu}$-invariants for sequences of lenght less than or equal to 5. Stallings [13] conjectured that there is a relationship between the $\bar{\mu}$ invariants and Massey products. Porter [10] and Turaev [16] were the first to prove this. The proof of Theorem 3.1 utilizes a refined expression of $\Delta$ and several lemmas. As above, let $G=\pi_{1}\left(S^{3}-L\right)$. We refer to the presentation $\left\langle x_{1}, \ldots, x_{n} \mid\left[x_{1}, v_{1 q}\right], \ldots,\left[x_{n}, v_{n q}\right]\right\rangle$ of $G / G_{q}$ described in $\S 2$. Let $I=\left(i_{1}, \ldots, i_{q}\right)$, and $r>0$.

Definition 3.2. For $I=\left(i_{1}, i_{2}\right)$, define $\Delta^{\prime}(I)=0$. For $q>2$, define $\Delta^{\prime}(I)=$ $\operatorname{gcd}\left\{m\left(t_{1}, \ldots, t_{l}: v_{t_{l+1} q+r}\right) \mid\left(t_{1}, \ldots, t_{l+1}\right)\right.$ is a proper subsequence of $\left.I\right\}$.

Lemma 3.3. For all $I, \Delta(I)=\Delta^{\prime}(I)$.

Proof. Induct on $q$. For $q=2$, the lemma is true by definition. Assume the lemma for indexing sequences of length less than $q$. Suppose $I=\left(i_{1}, \ldots, i_{q}\right)$. $\Delta(I)=\operatorname{gcd}\left\{m\left(s_{a}, \ldots, s_{b}: v_{s_{b+1} q+r}\right) \mid\left(s_{a}, \ldots, s_{b+1}\right)\right.$ is a cyclic permutation of a proper subsequence of $\left.\left(i_{1}, \ldots, i_{q}\right)\right\}$

$=\operatorname{gcd}\left\{\bar{\mu}\left(s_{1}, \ldots, s_{q-1}\right), \Delta\left(s_{1}, \ldots, s_{q-1}\right) \mid\left(s_{1}, \ldots, s_{q-1}\right)\right.$ is a cyclic permutation of a subsequence of $\left.\left(i_{1}, \ldots, i_{q}\right)\right\}$

$=\operatorname{gcd}\left\{\bar{\mu}\left(t_{1}, \ldots, t_{q-1}\right), \Delta^{\prime}\left(t_{1}, \ldots, t_{q-1}\right) \mid\left(t_{1}, \ldots, t_{q-1}\right)\right.$ is a subsequence of $\left.I\right\}$ $=\Delta^{\prime}(I)$.

Lemma 3.4. 1. If $i_{1}=i_{2}=\cdots=i_{q}$ then $m\left(I:\left[x_{j}, v_{j q+r}\right]\right)=0$. 
2. Otherwise,

$m\left(I:\left[x_{j}, v_{j q+r}\right]\right)=\sum_{\substack{\left(I_{1}, I_{2}, I_{3}, I_{4}\right)=I \\ I_{3} \neq \varnothing}} m\left(I_{1}: x_{j}^{-1}\right) m\left(I_{2}: v_{j q+r}^{-1}\right) m\left(I_{3}: x_{j}\right) m\left(I_{4}: v_{j q+r}\right)$. Proof. Suppose $i_{1}=i_{2}=\cdots=i_{q}=i$. As the sum of the exponents of $x_{i}$ in $\left[x_{j}, v_{j q+r}\right]$ is zero, $m\left(I:\left[x_{j}, v_{j q+r}\right]\right)=0$.

Statement 2 follows from $m\left(I:\left[x_{j}, v_{j q+r}\right]\right)=\sum_{\left(I_{1}, I_{2}, I_{3}, I_{4}\right)=I} m\left(I_{1}: x_{j}^{-1}\right) m\left(I_{2}: v_{j q+r}^{-1}\right) m\left(I_{3}: x_{j}\right) m\left(I_{4}: v_{j q+r}\right)$.

The sum of the terms with $I_{3}=\varnothing$ is

as the indices are not all identical.

$$
m\left(I: x_{j}^{-1} v_{j q+r}^{-1} v_{j q+r}\right)=m\left(I: x_{j}^{-1}\right)=0
$$

Lemma 3.5. Let $1 \leq k \leq q$, and let $\left(s_{1}, \ldots, s_{k}\right)$ be a subsequence of $I$.

Proof.

$$
m\left(s_{1}, \ldots, s_{k-1}: v_{s_{k} q+r}\right) \equiv-m\left(s_{1}, \ldots, s_{k-1}: v_{s_{k} q+r}^{-1}\right) \quad(\bmod \Delta(I)) .
$$

$$
\begin{aligned}
0 & =m\left(s_{1}, \ldots, s_{k-1}: v_{s_{k} q+r} v_{s_{k} q+r}^{-1}\right) \\
& =\sum_{\left(I_{1}, I_{2}\right)=\left(s_{1}, \ldots, s_{k-1}\right)} m\left(I_{1}: v_{s_{k} q+r}\right) m\left(I_{2}: v_{s_{k} q+r}^{-1}\right) \\
& =m\left(s_{1}, \ldots, s_{k-1}: v_{s_{k} q+r}\right)+m\left(s_{1}, \ldots, s_{k-1}: v_{s_{k} q+r}^{-1}\right) \quad(\bmod \Delta(I)) .
\end{aligned}
$$

Lemma 3.6. If $k>1$ and $i_{k} \neq i_{q}$, then

$$
m\left(i_{k+1}, \ldots, i_{q}: v_{i_{k} q+r}\right) \equiv 0 \quad(\bmod \Lambda(I)) .
$$

Proof. Let $I_{k}^{q}$ denote the subsequence $\left(i_{k}, \ldots, i_{q}\right)$ of $I$. Induct on the number $n$ of indices $i_{t}$ for $k+1 \leq t \leq q$, such that $i_{t}=i_{k}$. If $n=0$, then by Lemma $3.4(2)$,

In general:

$$
m\left(I_{k+1}^{q}: v_{i_{k} q+r}\right)=m\left(I_{k}^{q}:\left[x_{i_{k}}, v_{i_{k} q+r}\right]\right)=0 \quad(\bmod \Lambda(I))
$$

$$
\begin{aligned}
0 \equiv & m\left(I_{k}^{q}:\left[x_{i_{k}}, v_{i_{k} q+r}\right]\right)(\bmod \Lambda(I)) \\
= & {\left[\sum_{\substack{\left(I_{1}, I_{2}, I_{3}, I_{4}\right)=I_{k}^{q} \\
I_{3} \neq \varnothing,\left(I_{1}, I_{2}\right) \neq \varnothing}} m\left(I_{1}: x_{i_{k}}^{-1}\right) m\left(I_{2}: v_{v_{i_{k}} q+r}^{-1}\right) m\left(I_{3}: x_{i_{k}}\right) m\left(I_{4}: v_{i_{k} q+r}\right)\right] } \\
& +m\left(i_{k+1}, \ldots, i_{q}: v_{i_{k} q+r}\right) .
\end{aligned}
$$

Since $i_{q} \neq i_{k}, I_{4}$, in the above sum, is not empty. Furthermore, $I_{3}=\left(i_{\alpha}\right)$, and $i_{\alpha}=i_{k}$. Thus by induction,

$$
\sum_{\substack{\left(I_{1}, I_{2}, I_{3}, I_{4}\right)=I_{k} \\ I_{3} \neq \varnothing,\left(I_{1}, I_{2}\right) \neq \varnothing}} m\left(I_{1}: x_{i_{k}}^{-1}\right) m\left(I_{2}: v_{i_{k} q+r}^{-1}\right) m\left(I_{3}: x_{i_{k}}\right) m\left(I_{4}: v_{i_{k} q+r}\right) \equiv 0
$$


Thus

$$
m\left(i_{k+1}, \ldots, i_{q}: v_{i_{k} q+r}\right) \equiv 0 \quad(\bmod \Lambda(I)) .
$$

Lemma 3.7. 1. If $j \neq i_{1}$, and $j \neq i_{q}$, then $m\left(I:\left[x_{j}, v_{j q+r}\right]\right) \equiv 0(\bmod \Lambda(I))$.

2. $m\left(I:\left[x_{i_{1}}, v_{i_{1} q+r}\right]\right) \equiv-m\left(I:\left[x_{i_{q}}, v_{i_{q} q+r}\right]\right)(\bmod \Lambda(I))$.

Proof. Suppose $j \neq i_{1}$, and $j \neq i_{q}$. Invoking Lemma 3.4,

$$
m\left(I:\left[x_{j}, v_{j q+r}\right]\right)=\sum_{\substack{\left(I_{1}, I_{2}, I_{3}, I_{4}\right)=I \\ I_{3} \neq \varnothing}} m\left(I_{1}: x_{j}^{-1}\right) m\left(I_{2}: v_{j q+r}^{-1}\right) m\left(I_{3}: x_{j}\right) m\left(I_{4}: v_{j q+r}\right) .
$$

Since $j \neq i_{q}, I_{3} \neq\left(i_{q}\right)$, and thus $I_{4} \neq \varnothing$. Since $j \neq i_{1}, I_{3} \neq\left(i_{1}\right)$, and thus, by Lemma 3.6, $m\left(I_{4}: v_{j q+r}\right) \equiv 0(\bmod \Lambda(I))$. Therefore, $m\left(I:\left[x_{j}, v_{j q+r}\right]\right) \equiv 0$ $(\bmod \Lambda(I))$.

Statement 2 follows from statement 1 and two additional facts.

1. $\left\langle\sigma(I), \omega_{j}\right\rangle$ is the residue of $(-1)^{q+1} m\left(I:\left[x_{j}, v_{j q+r}\right]\right)$.

2. $\omega_{1}+\cdots+\omega_{n}=0$.

Proof of Theorem 3.1. We show that $\Delta(I) \mid \Lambda(I)$. Lemma 3.7 implies that $\Lambda(I)=$ $\operatorname{gcd}\left\{m\left(S:\left[x_{s_{k}}, v_{s_{k} q+r}\right]\right) \mid S=\left(s_{1}, \ldots, s_{k}\right)\right.$ is a proper subsequence of $I$, and $\left.s_{1} \neq s_{k}\right\}$.

$$
\begin{aligned}
m(S & \left.:\left[x_{s_{k}}, v_{s_{k} q+r}\right]\right) \\
& =\sum_{\substack{\left(I_{1}, I_{2}, I_{3}, I_{4}\right)=S \\
I_{3} \neq \varnothing}}\left[m\left(I_{1}: x_{s_{k}}^{-1}\right) m\left(I_{2}: v_{s_{k} q+r}^{-1}\right) m\left(I_{3}: x_{s_{k}}\right) m\left(I_{4}: v_{s_{k} q+r}\right)\right] .
\end{aligned}
$$

For each nonzero term of the sum, $I_{1}=\varnothing$, and $I_{2} \neq \varnothing$. Thus, by Lemma 3.5, $m\left(S:\left[x_{s_{k}}, v_{s_{k} q+r}\right]\right) \equiv 0(\bmod \Delta(I))$. Thus $\Delta(I)$ divides $\Lambda(I)$.

Proof that $\left\langle\sigma(I), \omega_{i_{q}}\right\rangle$ projects onto $(-1)^{q} \bar{\mu}(I) . h: H_{2}(G)-(N \cap F \sharp F) / F \sharp N$ is the Hopf isomorphism, and $h\left(\omega_{j}\right)$ is the class represented by $-w_{j r_{j}}$. See presentation (3) above. Thus

$$
\begin{aligned}
(-1)^{q}\left\langle\sigma(I), \omega_{i_{q}}\right\rangle & =-m\left(I:\left[x_{i_{q}}, v_{i_{q} q+r}\right]\right) \\
& =-\sum_{\substack{\left(I_{1}, I_{2}, I_{3}, I_{4}\right)=I \\
I_{3} \neq \varnothing}} m\left(I_{1}: x_{i_{q}}^{-1}\right) m\left(I_{2}: v_{i_{q} q+r}^{-1}\right) m\left(I_{3}: x_{i_{q}}\right) m\left(I_{4}: v_{i_{q} q+r}\right) .
\end{aligned}
$$

Since $i_{1} \neq i_{q}$, in each nonzero term of the above sum $I_{1}=\varnothing$, and $I_{2} \neq \varnothing$. If $I_{2} \neq\left(i_{1}, \ldots, i_{q-1}\right)$, then by Lemma 3.5, $m\left(I_{2}: v_{i_{q} q+r}^{-1}\right) \equiv 0(\bmod \Delta(I))$. Thus

$$
\begin{aligned}
(-1)^{q}\left\langle\sigma(I), \omega_{i_{q}}\right\rangle & \equiv-m\left(i_{1}, \ldots, i_{q-1}: v_{i_{q} q+r}^{-1}\right) \quad(\bmod \Delta(I)) \\
& \equiv m\left(i_{1}, \ldots, i_{q-1}: v_{i_{q} q+r}\right) \quad(\bmod \Delta(I)) \\
& =\bar{\mu}(I) .
\end{aligned}
$$

Proof that $\Delta(I)=\Lambda(I)$ if the indices are distinct. Suppose $S=\left(s_{1}, \ldots, s_{k}\right)$ is a subsequence of $I=\left(i_{1}, \ldots, i_{q}\right)$, and for all $\alpha \neq \beta, i_{\alpha} \neq i_{\beta}$. Then, $m\left(s_{1}, \ldots, s_{k-1}: v_{s_{k} q+r}\right)=-m\left(s_{1}, \ldots, s_{k}:\left[x_{s_{k}}, v_{s_{k} q+r}\right]\right)$, and thus $\Lambda(I) \mid \Delta(I)$. 
The present example shows that the $\sigma$ invariants may distinguish links which are not distinguished by the $\bar{\mu}$-invariants. Refer to Figures 1 and 2 below. The curves, $a$ and $b$, in Figures 1 and 2 are $V_{1} \cap V_{2}$ and $V_{1} \cap V_{3}$ respectively. Let $L=K_{1} \cup K_{2} \cup K_{3} \cup K_{4}$, and let $L^{\prime}=K_{1} \cup K_{2} \cup K_{3} \cup K_{4}^{\prime}$. One shows that

1. The $\sigma$ and $\bar{\mu}$ invariants, with distinct indices, are zero for each of these links.

2. Both of these links have $\bar{\mu}(1,2,3,1)=1$, and therefore, $\bar{\mu}(1,2,3,1,4)$ is totally indeterminate.

3. Each of these links has

$$
\Lambda(1,2,3,1,4)=0,
$$

and the invariants, $\sigma(1,2,3,1,4)\left(w_{4}\right)$, of $L$ and $L^{\prime}$ are 1 and 0 respectively.

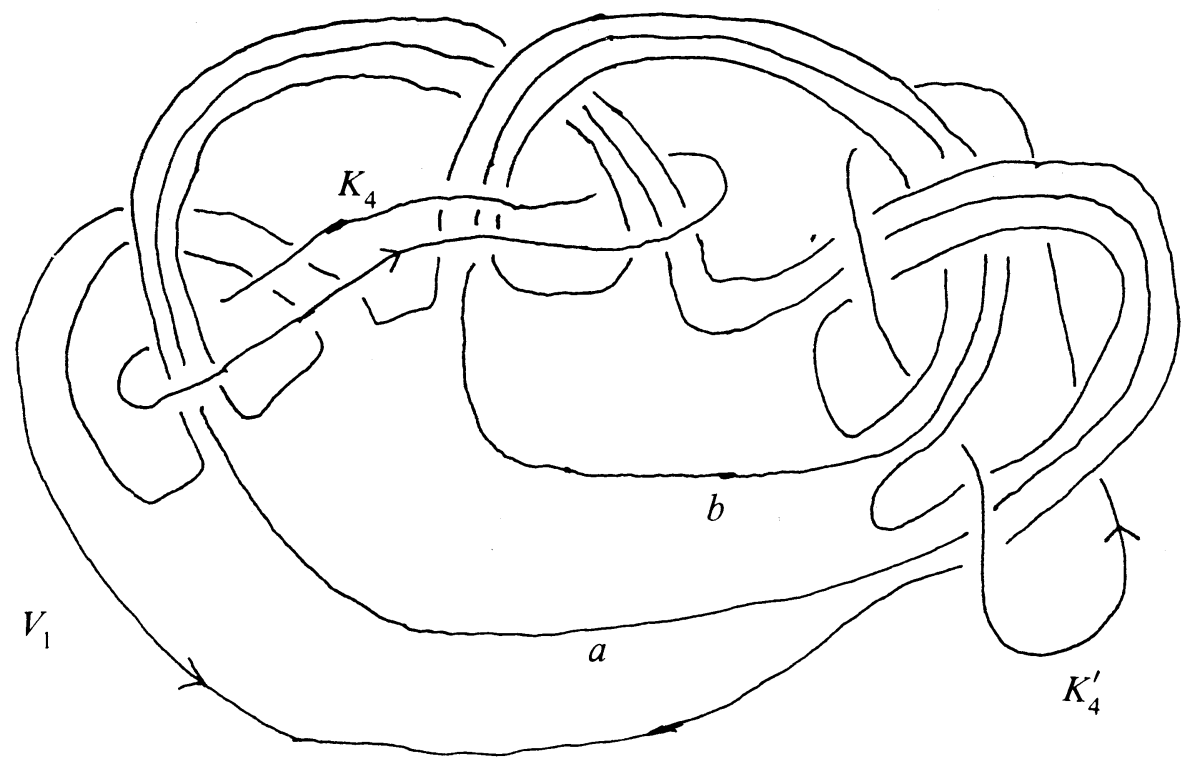

FigURE 1. $K_{1}=\partial V_{1} ; a=V_{1} \cap V_{2} ; b=V_{1} \cap V_{3}$

\section{Additional invariants}

In this section we show that for two component links certain of the $T_{V}$, using the appropriate group, are independent of the basing $V . L_{V}$, for these invariants, only depends upon the linking number of the components. If this linking number is zero, $L_{V}=0$, and these invariants may be related or equivalent to the Sato-Levine-Cochran invariants $[1,2,12]$. The invariants are defined using the fundamental group of the manifold obtained by surgery on one of the components.

Let $L$ be a link in $S^{3}$ with components $K_{1}$ and $K_{2}$. Let $W$ be the complement of $K_{2}$ in the manifold obtained by zero-framed surgery on $K_{1}$. Let $d=\left|\mathrm{l}\left(K_{1}, K_{2}\right)\right|$, and let $\mathscr{G}=\pi_{1}(W, w)$. 


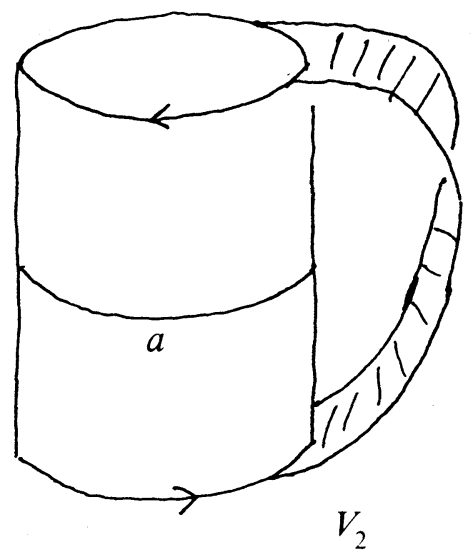

FIGURE 2. $K_{2}=\partial V_{2}$

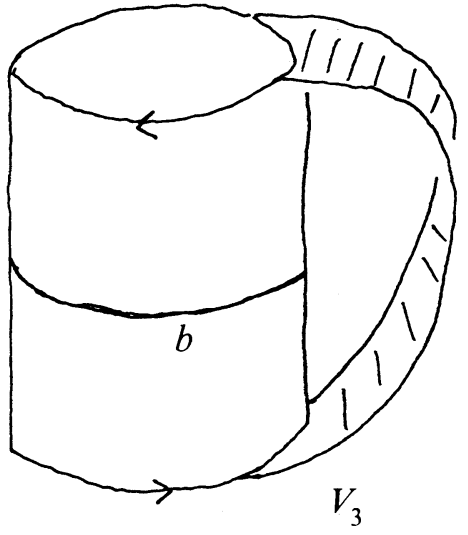

$K_{3}=\partial V_{3}$

Proposition 4.1. Let $V=\left\{v_{1}, v_{2}\right\} \subset \mathscr{G}$ be a collection of meridians of $L$. Then, $V$ satisfies $M(d)$.

Proof. Using the presentation (3) of $\pi_{1}\left(S^{3}-L\right)$ described above, such that for $i=1$ or $2, p\left(x_{i 1}\right)=v_{i}$, one sees that $\mathscr{G} \cong\left\langle x_{i j}\right| v_{1 r_{1}}, w_{s t} ; 1 \leq i \leq 2,1 \leq$ $\left.j \leq r_{i}, 1 \leq s \leq 2,1 \leq t \leq r_{i},(s, t) \neq\left(1, r_{1}\right)\right\rangle$, and that $v_{1 r_{1}}$ represents a longitude which has linking number zero with $K_{1}$. Let $v_{i q}=n_{q}\left(v_{i r_{i}}\right)$. Milnor's homomorphisms, $n_{q}: F \rightarrow A$, show that

$$
\mathscr{G} \mid \mathscr{G}_{q} \cong\left\langle x_{1}, x_{2} \mid v_{1 q},\left[x_{2}, v_{2 q}\right], A_{q}\right\rangle .
$$

Let $\omega \in A$, then $\omega \in A \sharp_{d} A \Leftrightarrow \forall 1 \leq j \leq 2, d \mid m(j: \omega)$ [4]. As $m\left(j: v_{1 q}\right)=$ $\operatorname{lk}\left(K_{j}, K_{1}\right), v_{1 q} \in A \sharp_{d} A$. Thus conditions 1 and 2 are satisfied. We verify 3 . Let $\lambda$ be the algebraic number of crossings of $K_{1}$ by itself in the projection of $L$. One may choose

Let

$$
v_{1 r_{1}}=x_{11}^{-\lambda} x_{\alpha_{1} \beta_{1}}^{\varepsilon_{1}} \cdots x_{\alpha_{r_{1}} \beta_{r_{1}}}^{\varepsilon_{r_{1}}}
$$

$$
\zeta_{1}=v_{1 r_{1}} \prod_{\beta_{j} \neq 1}\left(w_{\alpha_{j}\left(\beta_{j}-1\right)}\right)^{\varepsilon_{j}} .
$$

$\zeta_{1} \in F \sharp_{d} F$, and $n_{q}\left(\zeta_{1}\right) \equiv v_{1 q}\left(\bmod A_{q}\right)$. Thus $V$ satisfies $M(d)$.

For each $I \in I(2)$ and $C \in\left(H^{1}(W)\right)^{q}$, Theorems 1.7 and 1.11 provide invariants, $\sigma(I) \in H^{2}(\mathscr{G}, \mathbf{Z} / \Lambda(I))$ and $T_{V}(C) \in H^{2}\left(\mathscr{G}, \mathbf{Z} / L_{V}(C)\right)$.

From the exact homology sequence of the pair $(W, X), X=S^{3}-\operatorname{nbd}(L)$, one sees that $H_{2}(W ; \mathbf{Z} / d) \cong H_{2}(W, X ; \mathbf{Z} / d)$, and thus, $H_{2}(W ; \mathbf{Z} / d) \equiv \mathbf{Z} / d$. Let $\hat{\omega} \in H_{2}(W ; \mathbf{Z} / d)$ be the class represented by $D^{2} \cup \Sigma$, where $D^{2}$ is the core of the attached handle, and $\Sigma$ is a mod- $d$ Seifert surface for $K_{1}$ in $X$. Since $L$ has only two components, $\hat{\omega}$ is independent of the choice of the mod- $d$ Seifert surface, and it generates $H_{2}(W ; \mathbf{Z} / d)$. Under the Hopf homomorphisms utilized above, $\hat{\omega}$ corresponds to the class represented by $\zeta_{1}$. Let $\omega$ be the corresponding element of $H_{2}(\mathscr{G}, \mathbf{Z} / d)$. 
Certain of the invariants $T_{V}$ are independent of $V$. For each positive integer $k$ let $I_{k}$ be the sequence

$$
(2, \underbrace{1, \ldots, 1}_{2 k-1 \text { 1's }}, 2) \text {. }
$$

Theorem 4.2. 1 .

$$
L_{V}\left(I_{k}\right)=\operatorname{gcd}\left\{\left(\begin{array}{l}
d \\
j
\end{array}\right) \mid 1 \leq j \leq \min \{d, 2 k\}\right\} .
$$

Thus, if the linking number is zero, $L_{V}\left(I_{k}\right)=0$, while if $d \neq 0$ and $2 k \geq$ $d, L_{V}\left(I_{k}\right)=1$, and $T_{V}\left(I_{k}\right)$ is totally indeterminate.

2. $T_{V}\left(I_{k}\right)$ is independent of $V$.

The proof quotes the following lemma.

Lemma 4.3. Let $E$ be the free group $\left\langle x_{1}, \ldots, x_{m}\right\rangle$. For each $w \in E$ and each positive integer $s$ define

$$
\Theta(s, w)=\operatorname{gcd}\{m(\underbrace{1, \ldots, 1}_{r \text { 1's }}: w) \mid 1 \leq r \leq s\} .
$$

Then, $\Theta(s, w)=\Theta\left(s, w^{-1}\right)$.

Proof. Induct on $s$. Let $s=1 . m(1: w)=-m\left(1: w^{-1}\right)$. Assume the result for $s<q$, and let

$$
I=(\underbrace{1, \ldots, 1}_{q \text { l's }}) \text {. }
$$

$$
\begin{aligned}
0= & m\left(I: w w^{-1}\right) \\
= & m(I: w)+m\left(I: w^{-1}\right)+\sum_{\substack{\left(I_{1}, I_{2}\right)=I \\
I_{1} \neq \varnothing, I_{2} \neq \varnothing}} m\left(I_{1}: w\right) m\left(I_{2}: w^{-1}\right) \\
& \equiv m(I: w)+m\left(I: w^{-1}\right) \quad(\bmod \Theta(q-1, w)) \\
\Theta(q, w) & =\operatorname{gcd}\{\Theta(q-1, w), m(I: w)\} \\
& =\operatorname{gcd}\left\{\Theta\left(q-1, w^{-1}\right), m\left(I: w^{-1}\right)\right\} \quad \text { (by induction and (5)) } \\
& =\Theta\left(q, w^{-1}\right) .
\end{aligned}
$$


Proof of 4.2.1.

$$
\begin{aligned}
& L_{V}\left(I_{k}\right)=\operatorname{gcd}\left\{m\left(J: v_{12 k+1+r}\right) \mid J=(2, \underbrace{1, \ldots, 1}_{r \text { l's }}),(\underbrace{1, \ldots, 1}_{r \text { l's }}, 2),\right. \\
& \text { or }(\underbrace{1, \ldots, 1}_{r \text { l's }}) \text {, and } 0 \leq r \leq 2 k-1\} \\
& =\Delta(\underbrace{1, \ldots, 1}_{2 k+1 \text { l's }}, 2) \\
& =\operatorname{gcd}\left\{m(\underbrace{1, \ldots, 1}_{r \text { l's }}: v_{22 k+1+r}) \mid 1 \leq r \leq 2 k\right\} \quad(\text { Lemma 3.3) } \\
& =\operatorname{gcd}\left\{m(\underbrace{1, \ldots, 1}_{r \text { l's }}: x_{1}^{1 \mathrm{k}\left(K_{1}, K_{2}\right)}) \mid 1 \leq r \leq 2 k\right\} \\
& =\operatorname{gcd}\left\{m(\underbrace{1, \ldots, 1}_{r \text { 1's }}: x_{1}^{d}) \mid 1 \leq r \leq 2 k\right\} \quad(\text { Lemma 4.3). }
\end{aligned}
$$

The statement follows as

$$
m(\underbrace{1, \ldots, 1}_{r \text { l's }}: x_{1}^{d})=\left(\begin{array}{c}
d \\
r
\end{array}\right) .
$$

Definition 4.4. For any basing $V$ of $L, L\left(I_{k}\right)=L_{V}\left(I_{k}\right)$.

The proof of statement 2 requires a definition and some lemmas.

Definition 4.5. Let $x=\mathscr{G}$, and let $\Upsilon: \mathscr{G} \rightarrow \overline{\mathbf{U}}\left(\mathbf{Z} / L\left(I_{k}\right), 2 k+2\right)$ be a homomorphism. $\Upsilon$ is internally zero on $x$ if $\Upsilon_{i j}(x)=0$ for all $2 \leq i<j-1 \leq 2 k$.

Lemma 4.6. Let $x$ and $y \in \mathscr{G}$ be meridians of $K_{1}$ and $K_{2}$ respectively. If $\Upsilon$, a defining system for $I_{k}$, is internally zero on $x$ and semizero on $\{y\}$, then for all $g \in G, \Upsilon$ is internally zero on $\mathrm{gxg}^{-1}$.

Proof. Let $A$ be the free group $\left\langle x_{1}, x_{2}\right\rangle$. For each $r>0$, there is a diagram

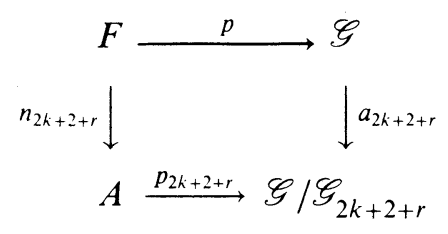

such that if $x^{\prime}=a_{2 k+2+r}(x)$, and $y^{\prime}=a_{2 k+2+r}(y), p_{2 k+2+r}\left(x_{1}\right)=x^{\prime}$, and $p_{2 k+2+r}\left(x_{2}\right)=y^{\prime}$. Choose $(i, j)$ such that $2 \leq i<j-1 \leq 2 k$. Let $\Upsilon^{\prime}$ : $\mathscr{G} / \mathscr{G}_{2 k+2+r} \rightarrow \overline{\mathbf{U}}\left(\mathbf{Z} / L\left(I_{k}\right), 2 k+2\right)$ be the induced homomorphism. The argument below hinges upon the fact that, for such $(i, j), \Upsilon_{i j}(x)=\Upsilon_{i j}(y)=0$, and thus $\Upsilon_{i j}^{\prime}\left(x^{\prime}\right)=\Upsilon_{i j}^{\prime}\left(y^{\prime}\right)=0$. Let $\tilde{g} \in p^{-1}(g)$, and let $\hat{g}=n_{2 k+2+r}(\tilde{g})$. Let

$$
\tilde{\Upsilon}^{\prime}: A \rightarrow \mathbf{U}\left(\mathbf{Z} / L\left(I_{k}\right), 2 k+2\right)
$$


be a homomorphism such that the diagram below commutes.

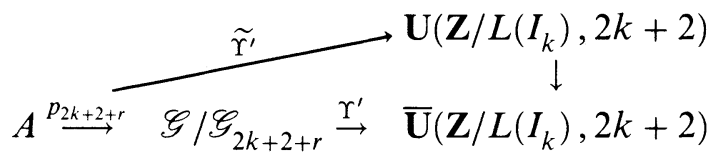

$$
\begin{aligned}
& \Upsilon_{i j}\left(g x g^{-1}\right)=\Upsilon_{i j}^{\prime}\left(a_{2 k+2+r}\left(g \times g^{-1}\right)\right) \\
& =\sum_{s=1}^{j-i} \sum_{c_{1}, \ldots, c_{s} \in S_{i j}} \sum_{I \in I(2)_{s}}\left(\widetilde{\Upsilon}_{i i+c_{1}}^{\prime} \cdots \widetilde{\Upsilon}_{j-c_{s} j}^{\prime}\right)(I) m\left(I: \hat{g} x_{1} \hat{g}^{-1}\right) \\
& =(-1)^{j-i} m(\underbrace{1, \ldots, 1}_{j-i \text { l's }}: \hat{g} x_{1} \hat{g}^{-1}) \\
& =(-1)^{j-i} m\left(1, \ldots, 1: x_{1}\right) \\
& =0 \text {. }
\end{aligned}
$$

Lemma 4.7. Let $V=\{x, y\}$ as above. If $\Upsilon$, a defining system for $I_{k}$, is internally zero on $x$ and zero on $\{y\}$, then $\langle[\Upsilon], \check{\omega}\rangle=\left\langle T_{V}\left(I_{k}\right), \check{\omega}\right\rangle$.

Proof. Let $\Upsilon, \Upsilon^{\prime}$, and $\widetilde{\Upsilon^{\prime}}$ be as defined above, and suppose that $\Upsilon$ is internally zero on $x$ and zero on $\{y\}$.

$$
\begin{aligned}
\langle[\Upsilon], \check{\omega}\rangle & =\tilde{\Upsilon}_{12 k+2}^{\prime}\left(v_{12 k+2+r}\right) \\
& =\sum_{s=1}^{2 k+1} \sum_{c_{1}, \ldots, c_{s} \in S_{12 k+2}} \sum_{I \in I(2)_{s}}\left(\widetilde{\Upsilon}_{11+c_{1}}^{\prime} \ldots \widetilde{\Upsilon}_{2 k+2-c_{s} 2 k+2}^{\prime}\right)(I) m\left(I: v_{12 k+2+r}\right) \\
& =-m(2, \underbrace{1, \ldots, 1}_{2 k-11 \text { l's }}, 2: v_{12 k+2+r}) \\
& =\left\langle T_{V}\left(I_{k}\right), \check{\omega}\right\rangle .
\end{aligned}
$$

This argument uses the fact that

$$
\left.\widetilde{\Upsilon}_{11+c_{1}}^{\prime} \cdots \widetilde{\Upsilon}_{2 k+2-c_{s} 2 k+2}^{\prime}\right)(I) m\left(I: v_{12 k+2+r}\right) \equiv 0 \quad\left(\bmod L\left(I_{k}\right)\right)
$$

unless $s=2 k+1,\left(c_{1}, \ldots, c_{2 k+1}\right)=(1, \ldots, 1)$, and $I=(2,1, \ldots, 1,2)$, which we explain. Suppose $s=1 . m\left(1: v_{12 k+2+r}\right)=0$ as the self-linking number is zero, and $m\left(2: v_{1} 2 k+2+r\right)=1 \mathrm{k}\left(K_{1}, K_{2}\right)=d$. Suppose

$$
\left(\widetilde{\Upsilon}_{11+c_{1}}^{\prime} \cdots \widetilde{\Upsilon}_{2 k+2-c_{s} 2 k+2}^{\prime}\right)(I) m\left(I: v_{12 k+2+r}\right) \not \equiv 0 \text {. }
$$

Each of the numbers, $m\left(1, \ldots, 1: v_{12 k+2+r}\right), m\left(2,1, \ldots, 1: v_{12 k+2+r}\right)$, and $m\left(1, \ldots, 1,2: v_{12 k+2+r}\right)$, is congruent to zero $\bmod L\left(I_{k}\right)$. Since $\widetilde{\Upsilon}^{\prime}$ is zero on $\left\{x_{2}\right\}, c_{1}=1$, and $c_{s}=1$. For $2 \leq i<j-1<2 k+1, \tilde{\Upsilon}_{i j}^{\prime}\left(x_{1}\right)=0$. Thus, for $1<t<s, c_{t}=1$. As $c_{1}+c_{2}+\cdots+c_{s}=2 k+2$, the claim follows.

Lemma 4.8. Let $\left\{m_{1}, m_{2}\right\}$ be a collection of meridians of $L$ in $\mathscr{G}$, and let $\Upsilon$ be a defining system for $I_{k}$ which is zero on $\left\{m_{1}, m_{2}\right\}$ as above. Let $m_{2}^{+}=$ $m_{1} m_{2} m_{1}^{-1}$, and let $m_{2}^{-}=m_{1}^{-1} m_{2} m_{1}$. Let $\Upsilon_{+}$and $\Upsilon_{-}$be defining systems for 
$I_{k}$ which are zero on $\left\{m_{1}, m_{2}^{+}\right\}$, and $\left\{m_{1}, m_{2}^{-}\right\}$respectively. Then $\left\langle\left[\Upsilon_{+}\right], \omega\right\rangle=$ $\langle[\Upsilon], \omega\rangle=\left\langle\left[\Upsilon_{-}\right], \omega\right\rangle$.

Proof.

$$
\Upsilon_{+}^{\prime}: \mathscr{G} / \mathscr{G}_{2 k+2+r} \rightarrow \overline{\mathbf{U}}\left(\mathbf{Z} / L\left(I_{k}\right), 2 k+2\right)
$$

and

$$
\Upsilon_{-}^{\prime}: \mathscr{G} / \mathscr{G}_{2 k+2+r} \rightarrow \overline{\mathbf{U}}\left(\mathbf{Z} / L\left(I_{k}\right), 2 k+2\right),
$$

are the induced defining systems. Let $p_{2 k+2+r}: A \rightarrow \mathscr{G} / \mathscr{G}_{2 k+2-r}$ be as defined above, and let $\widetilde{\Upsilon}_{+}^{\prime}$ and $\widetilde{\Upsilon}_{-}^{\prime}$ be homomorphisms such that the following diagrams commute.

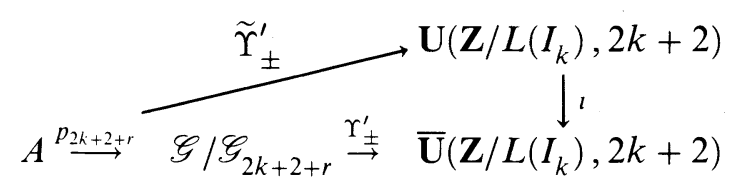

Claim. 1. $\tilde{\Upsilon}^{\prime}\left(x_{1}\right)=\tilde{\Upsilon}_{+}^{\prime}\left(x_{1}\right)=\tilde{\Upsilon}_{-}^{\prime}\left(x_{1}\right)$.

2. For $2 \leq i<j-1<2 k+1, \widetilde{\Upsilon}_{ \pm i j}^{\prime}\left(x_{2}\right)=0$.

3. For $3 \leq j \leq 2 k+1$,

$$
\tilde{\Upsilon}_{+1 j}^{\prime}\left(x_{2}\right)=\left\{\begin{array}{ll}
1, & \text { if } j=3, \\
0, & \text { otherwise, }
\end{array} \quad \widetilde{\Upsilon}_{-1 j}^{\prime}\left(x_{2}\right)=-1 .\right.
$$

4. For $2 \leq l \leq 2 k$,

$$
\tilde{\Upsilon}_{+l 2 k+2}^{\prime}\left(x_{2}\right)=-1, \quad \widetilde{\Upsilon}_{-l 2 k+2}^{\prime}\left(x_{2}\right)= \begin{cases}1, & \text { if } l=2 k \\ 0, & \text { otherwise } .\end{cases}
$$

Proof of 1. For $g \in \mathscr{G}$, let $\bar{g}=a_{2 k+2+r}(g)$. Statement 1 follows from the fact that $\Upsilon^{\prime}, \Upsilon_{+}^{\prime}$, and $\Upsilon_{-}^{\prime}$ are zero on $\overline{m_{1}}$.

Proof of 2. Let $\Phi_{22 k+1}: \overline{\mathbf{U}}\left(\mathbf{Z} / L\left(I_{k}\right), 2 k+2\right) \rightarrow \mathbf{U}\left(\mathbf{Z} / L\left(I_{k}\right), 2 k\right)$ be the homomorphism defined in $\S 1 . \overline{m_{2}}={\overline{m_{1}}}^{-1} \overline{m_{2}^{+}} \overline{m_{1}}$, and $\overline{m_{2}}=\overline{m_{1}}{\overline{m_{2}}}_{\overline{m_{1}}}^{-1}$. We state the argument for $\tilde{\Upsilon}_{+}^{\prime}$, and the argument for $\widetilde{\Upsilon}_{-}^{\prime}$ is similar. Let ID be the identity matrix.

$$
\begin{aligned}
& \Phi_{22 k+1} \circ l \circ \tilde{\Upsilon}_{+}^{\prime}\left(x_{2}\right)=\Phi_{22 k+1} \circ \Upsilon_{+}^{\prime}\left(\overline{m_{2}}\right) \\
& =\Phi_{22 k+1} \circ \Upsilon_{+}^{\prime}\left({\overline{m_{1}}}^{-1}{\overline{m_{2}^{+}}}_{m_{1}}\right) \\
& =\Phi_{22 k+1} \circ \Upsilon_{+}^{\prime}\left({\overline{m_{1}}}^{-1}\right) \cdot \Phi_{22 k+1} \circ \Upsilon_{+}^{\prime}\left(\overline{m_{2}^{+}}\right) \cdot \Phi_{22 k+1} \circ \Upsilon_{+}^{\prime}\left(\overline{m_{1}}\right) \\
& =\Phi_{22 k+1} \circ \Upsilon_{+}^{\prime}\left({\overline{m_{1}}}^{-1}\right) \cdot \operatorname{ID} \cdot \Phi_{22 k+1} \circ \Upsilon_{+}^{\prime}\left(\overline{m_{1}}\right) \\
& =\text { ID. }
\end{aligned}
$$


Proof of 3. Let $x_{2}^{+}=x_{1} x_{2} x_{1}^{-1}$ and $x_{2}^{-}=x_{1}^{-1} x_{2} x_{1} \cdot A$ is generated by both $\left\{x_{1}, x_{2}^{+}\right\}$and $\left\{x_{1}, x_{2}^{-}\right\}$. Since $x_{2}=x_{1}^{-1} x_{2}^{+} x_{1}$, and $x_{2}=x_{1} x_{2}^{-} x_{1}^{-1}$,

$$
\begin{aligned}
\widetilde{\Upsilon}_{+1 j}^{\prime}\left(x_{2}\right) & =(-1)^{j-1} m(2, \underbrace{1, \ldots, 1}_{j-21 ' s}: x_{1}^{-1} x_{2}^{+} x_{1}) \quad(\text { Lemma 1.5 }) \\
& = \begin{cases}1, & \text { if } j=3, \\
0, & \text { otherwise },\end{cases} \\
\widetilde{\Upsilon}_{-1 j}^{\prime}\left(x_{2}\right) & =(-1)^{j-1} m(2, \underbrace{1, \ldots, 1}_{j-21 \text { 's }}: x_{1} x_{2}^{-} x_{1}^{-1}) \\
& =(-1)^{j-1}(-1)^{j-2} \\
& =-1 .
\end{aligned}
$$

Proof of 4. For $2 \leq l \leq 2 k$,

$$
\begin{aligned}
\tilde{\Upsilon}_{+l 2 k+2}^{\prime}\left(x_{2}\right) & =(-1)^{l} m(\underbrace{1, \ldots, s}_{2 k+1-l 1}, 2: x_{1}^{-1} x_{2}^{+} x_{1}) \\
& =(-1)^{l}(-1)^{l-1} \\
& =-1, \\
\tilde{\Upsilon}_{-l 2 k+2}^{\prime}\left(x_{2}\right) & =(-1)^{l} m(\underbrace{1, \ldots, 1}_{2 k+1-l 1 \text { 's }}, 2: x_{1} x_{2}^{-} x_{1}^{-1}) \\
& = \begin{cases}1, & \text { if } l=2 k, \\
0, & \text { otherwise. }\end{cases}
\end{aligned}
$$

Proof of the lemma.

$$
\begin{aligned}
& \left\langle\left[\Upsilon_{-}\right], \omega\right\rangle=\widetilde{\Upsilon}_{-12 k+2 D}^{\prime}\left(v_{12 k+2+r}\right) \\
& =\tilde{\Upsilon}_{-12}^{\prime} \tilde{\Upsilon}_{-23}^{\prime} \cdots \tilde{\Upsilon}_{-2 k+12 k+2}^{\prime}\left(I_{k}\right) m\left(I_{k}: v_{12 k+2+r}\right) \\
& +\sum_{j=3}^{2 k+1}\left[\left[\widetilde{\Upsilon}_{-1 j}^{\prime} \widetilde{\Upsilon}_{-j j+1}^{\prime} \cdots \tilde{\Upsilon}_{-2 k+12 k+2}^{\prime}(2, \underbrace{1, \ldots, 1}_{2 k+1-j 1 \text { 's }}, 2)\right.\right. \\
& \left.\times m(2, \underbrace{1, \ldots, 1}_{2 k+1-j \text { l's }}, 2: v_{12 k+2+r})\right] \\
& +\left[\tilde{\Upsilon}_{-1 j-1}^{\prime} \tilde{\Upsilon}_{-j-1 j}^{\prime} \cdots \tilde{\Upsilon}_{-2 k-12 k}^{\prime} \tilde{\Upsilon}_{-2 k 2 k+2}^{\prime}(2, \underbrace{1, \ldots, 1}_{2 k+1-j 1 \text { 's }}, 2)\right. \\
& \left.\left.\times m(2, \underbrace{1, \ldots, 1}_{2 k+1-j \text { l's }}, 2: v_{12 k+2+r})\right]\right] \\
& +\widetilde{\Upsilon}_{-12 k+2}^{\prime}(1) m\left(1: v_{12 k+2+r}\right)+\widetilde{\Upsilon}_{-12 k+2}^{\prime}(2) m\left(2: v_{12 k+2+r}\right) \text {. }
\end{aligned}
$$


$m\left(j: v_{12 k+2+r}\right) \equiv 0\left(\bmod L\left(I_{k}\right)\right)$ for $j=1,2$, and

$$
\begin{aligned}
(-1)^{2 k+3-j} & =\widetilde{\Upsilon}_{-1 j}^{\prime} \tilde{\Upsilon}_{-j j+1}^{\prime} \ldots \tilde{\Upsilon}_{-2 k+12 k+2}^{\prime}(2, \underbrace{1, \ldots, 1}_{2 k+1-j 1 \text { 's }}, 2) \\
& =-\widetilde{\Upsilon}_{-1 j-1}^{\prime} \tilde{\Upsilon}_{-j-1 j}^{\prime} \ldots \widetilde{\Upsilon}_{-2 k-12 k}^{\prime} \widetilde{\Upsilon}_{-2 k 2 k+2}^{\prime}(2, \underbrace{1, \ldots, 1}_{2 k+1-j 1 \text { 's }}, 2) .
\end{aligned}
$$

Thus

$$
\begin{aligned}
\left\langle\left[\Upsilon_{-}\right], \omega\right\rangle & \equiv \widetilde{\Upsilon}_{-12}^{\prime} \widetilde{\Upsilon}_{-23}^{\prime} \cdots \widetilde{\Upsilon}_{-2 k+12 k+2}^{\prime}\left(I_{k}\right) m\left(I_{k}: v_{12 k+2+r}\right) \\
& =(-1)^{2 k+1} m\left(I_{k}: v_{12 k+2+r}\right) \\
& =\langle[\Upsilon], \omega\rangle .
\end{aligned}
$$

A similar argument establishes the corresponding result for $\left\langle\left[\Upsilon_{+}\right], \omega\right\rangle$.

Definition 4.9. Let $V=\left\{m_{1}, m_{2}\right\}$ and $\check{V}=\left\{\check{m}_{1}, \check{m}_{2}\right\}$ be basings of the link $L$. $V \sim \check{V}$ if $T_{V}\left(I_{k}\right)=T_{\check{V}}\left(I_{k}\right)$.

Lemma 4.10. Suppose $g$ is a loop in $W$, and $\operatorname{lk}\left(g, K_{1}\right)=0$. If $\Upsilon$ is a defining system for $I_{k}$ which is zero on $\left\{m_{1}, m_{2}\right\}$, then $\Upsilon$ is zero on $\left\{m_{1}, g m_{2} g^{-1}\right\}$. Proof. Let $w_{g} \in F$ be a word such that $p\left(w_{g}\right)=g$, and let $w_{g}^{\prime}=n_{2 k+2}\left(w_{g}\right)$. Suppose $1 \leq i<j \leq 2 k+2$, and $(i, j) \neq(1,2 k+2)$. For $I=\left(l_{1}, \ldots, l_{q}\right)$ and $1 \leq s \leq t \leq q$, let $I_{s}^{t}=\left(l_{s}, \ldots, l_{t}\right)$.

$$
\begin{aligned}
\Upsilon_{i j}\left(g m_{2} g^{-1}\right) & =\widetilde{\Upsilon}_{i j}^{\prime}\left(w_{g}^{\prime} x_{2} w_{g}^{\prime-1}\right) \\
& =(-1)^{j-i} m\left(I_{i}^{j-1}: w_{g}^{\prime} x_{2} w_{g}^{\prime-1}\right) \\
& =\sum_{\substack{\left(I_{1}, I_{2}, I_{3}\right)=I_{i}^{j-1} \\
I_{2}=2}} m\left(I_{1}: w_{g}^{\prime}\right) m\left(I_{2}: x_{2}\right) m\left(I_{3}: w_{g}^{\prime-1}\right) .
\end{aligned}
$$

If $i \neq 1$, and $j \neq 2 k+2$, then

$$
I_{i}^{j-1}=(\underbrace{1, \ldots, 1}_{j-i 1 \text { 's }}),
$$

and thus $m\left(I_{i}^{j-1}: w_{g}^{\prime} x_{2} w_{g}^{\prime-1}\right)=0$. If $i=1$, and $j \neq 2 k+2$, then

$$
I_{1}^{j-1}=(2, \underbrace{1, \ldots, 1}_{j-21 \text { 's }}) \text {, }
$$

and thus

$$
\begin{aligned}
m\left(I_{1}^{j-1}: w_{g}^{\prime} x_{2} w_{g}^{\prime-1}\right) & =m(\underbrace{1, \ldots, 1}_{j-21 ' s}: w_{g}^{\prime-1}) \\
& =m(\underbrace{1, \ldots, 1}_{j-21 ' s}: x_{1}^{-\operatorname{lk}\left(g, k_{1}\right)}) \\
& =0 .
\end{aligned}
$$


If $i \neq 1$, and $j=2 k+2$, then

$$
I_{i}^{2 k+1}=(\underbrace{1, \ldots, 1}_{2 k-i \text { l's }}, 2)
$$

and thus

$$
\begin{aligned}
m\left(I_{i}^{2 k+1}: w_{g}^{\prime} x_{2} w_{g}^{\prime-1}\right) & =m(\underbrace{1, \ldots, 1}_{2 k-i 1 \text { 's }}: w_{g}^{\prime}) \\
& =m(\underbrace{1, \ldots, 1}_{2 k-i 1 \text { 's }}: x_{1}^{\mathrm{lk}\left(g, k_{1}\right)}) \\
& =0 .
\end{aligned}
$$

Lemma 4.11. Let $V=\left\{m_{1}, m_{2}\right\}$ be a basing of $L$, and for $n \in \mathbf{Z}$, let $V_{n}=$ $\left\{m_{1}, m_{1}^{n} m_{2} m_{1}^{-n}\right\} . V \sim V_{n}$.

Proof. Induct on $n$. Lemma 4.8 covers the cases $n= \pm 1$. Let $\Upsilon_{n}$ be a defining system for $I_{k}$ which is zero on $V_{n}$. Then, for $n>0$,

$$
\begin{aligned}
\left\langle\left[\Upsilon_{n}\right], \omega\right\rangle & =\left\langle\left[\Upsilon_{n-1}\right], \omega\right\rangle \quad \text { (by Lemma 4.8) } \\
& =\left\langle\left[\Upsilon_{0}\right], \omega\right\rangle \quad \text { (by induction). }
\end{aligned}
$$

Thus $V \sim V_{n}$. The case $n<0$ follows similarly.

Proof of 4.2.2. Let $V=\left\{m_{1}, m_{2}\right\}$ and $\check{V}=\left\{\check{m}_{1}, \check{m}_{2}\right\}$ be basings of $L$. For some $g \in \mathscr{G}, \check{m}_{2}=g m_{2} g^{-1}$. Suppose $g$ is represented by the loop $\Gamma$, and let $n=\operatorname{lk}\left(\Gamma, K_{1}\right)$. Of course, $n$ depends only upon the homology class represented by $\Gamma$.

$$
\begin{aligned}
\left\{m_{1}, m_{2}\right\} & \sim\left\{\check{m}_{1}, m_{2}\right\} \quad(\text { by Lemmas } 4.6 \text { and } 4.7) \\
& \sim\left\{\check{m}_{1}, \check{m}_{1}^{n} m_{2} \check{m}_{1}^{-n}\right\} \quad(\text { by Lemma 4.11) } \\
& \sim\left\{\check{m}_{1}, \check{m}_{2}\right\} \quad(\text { by Lemma } 4.10)
\end{aligned}
$$

The invariants in the surgered manifold are compared with the invariants in the complement of the link. For $k>0$, let $I_{k}$ be as above, and let

$$
J_{k}=(2, \underbrace{1, \ldots, 1}_{2 k-11 \text { 's }}, 2,1) .
$$

Let $\omega_{1} \in H_{2}(G), \omega \in H_{2}(\mathscr{G})$, and $V=\left\{m_{1}, m_{2}\right\}$ be as defined above. We have

$$
T_{V}\left(J_{k}\right) \in H^{2}\left(G, \mathbf{Z} / L_{V}\left(J_{k}\right)\right), \quad \sigma\left(J_{k}\right) \in H^{2}\left(G, \mathbf{Z} / \Lambda\left(J_{k}\right)\right),
$$

and

$$
T\left(I_{k}\right) \in H^{2}\left(\mathscr{G}, \mathbf{Z} / L\left(I_{k}\right)\right)
$$

Theorem 4.12. 1. If $\operatorname{lk}\left(K_{1}, K_{2}\right)=0$, then $L\left(I_{k}\right)=0=L_{V}\left(J_{k}\right)$, and $T\left(I_{k}\right)(\omega)=$ $T_{V}\left(J_{k}\right)\left(\omega_{1}\right)$. In general $L\left(I_{k}\right) \mid L_{V}\left(J_{k}\right)$ and

$$
T\left(I_{k}\right)(\omega) \equiv-T_{V}\left(J_{k}\right)\left(\omega_{1}\right) \quad\left(\bmod L\left(I_{k}\right)\right)
$$


2. $\Lambda\left(J_{k}\right) \mid L\left(I_{k}\right)$ and

Proof of 4.12.1.

$$
\sigma\left(J_{k}\right)\left(\omega_{1}\right) \equiv-T\left(I_{k}\right)(\omega) \quad\left(\bmod \Lambda\left(J_{k}\right)\right) .
$$

$$
\begin{aligned}
L_{V}\left(J_{k}\right)=\operatorname{gcd}\left\{m(2, \underbrace{1, \ldots, 1}_{l \text { 's }}:\left[x_{1}, v_{12 k+2+r}\right]),\right. \\
\left.m(\underbrace{1, \ldots, 1}_{l \text { ''s }}, 2:\left[x_{1}, v_{12 k+2+r}\right]) \mid 0 \leq l \leq 2 k-1\right\} .
\end{aligned}
$$

Results analogous to Lemmas 3.4 and 3.5 and the proof of Theorem 4.2.1 show that $L\left(I_{k}\right) \mid L_{V}\left(J_{k}\right)$. If $\operatorname{lk}\left(K_{1}, K_{2}\right)=0, L\left(I_{k}\right)=0$, and thus, $L_{V}\left(J_{k}\right)=0$.

$$
\begin{aligned}
T_{V}\left(J_{k}\right)\left(\omega_{1}\right)=m(2, \underbrace{1, \ldots, 1}_{2 k-11 ' s}, 2,1: v_{12 k+2+r} x_{1} v_{12 k+2+r}^{-1} x_{1}^{-1}) \\
\quad=\sum_{\substack{\left(I_{1}, I_{2}, I_{3}, I_{4}\right)=J_{k} \\
I_{2} \neq \varnothing}} m\left(I_{1}: v_{12 k+2+r}\right) m\left(I_{2}: x_{1}\right) m\left(I_{3}: v_{12 k+2+r}^{-1}\right) m\left(I_{4}: x_{1}^{-1}\right) \\
\quad \equiv m(2, \underbrace{1, \ldots, 1}_{2 k-11 ', \ldots, 1}, 2: v_{12 k+2+r}) \quad\left(\bmod L\left(I_{k}\right)\right) \\
=-T\left(I_{k}\right)(\omega) .
\end{aligned}
$$

The proof of 2 utilizes the lemma below.

Lemma 4.13. For each $0 \leq s \leq 2 k-1$,

Proof.

$$
m(2, \underbrace{1, \ldots, 1}_{s \text { I's }}: v_{12 k+2+r}) \equiv 0 \quad\left(\bmod \Lambda\left(J_{k}\right)\right) .
$$

$$
\begin{aligned}
\Lambda\left(J_{k}\right)=\operatorname{gcd} & \left\{m(2, \underbrace{1, \ldots, 1}_{r \text { l's }}: v_{12 k+2+r} x_{1} v_{12 k+2+r}^{-1} x_{1}^{-1}),\right. \\
& m(2, \underbrace{1, \ldots, 1}_{l \text { l's }}, 2,1: v_{12 k+2+r} x_{1} v_{12 k+2+r}^{-1} x_{1}^{-1}), \\
& m(\underbrace{1, \ldots, 1}_{p \text { l's }}, 2: v_{12 k+2+r} x_{1} v_{12 k+2+r}^{-1} x_{1}^{-1}) \mid \\
0 & \leq r \leq 2 k, 0 \leq l \leq 2 k-2,0 \leq p \leq 2 k-1\} .
\end{aligned}
$$

Induct on $s$. Consider the case $s=0$. Let $r=1$.

$$
\begin{aligned}
0 & \equiv m\left(2,1: v_{12 k+2+r} x_{1} v_{12 k+2+r}^{-1} x_{1}^{-1}\right) \quad\left(\bmod \Lambda\left(J_{k}\right)\right) \\
& =m\left(2: v_{12 k+2+r}\right) .
\end{aligned}
$$

Assume the lemma is true for $0 \leq s<q \leq 2 k-1$. Let $r=q+1$, and let

$$
I=(2, \underbrace{1, \ldots, 1}_{r \text { 1's }}) \text {. }
$$




$$
\begin{aligned}
0 & \equiv m\left(I: v_{1} 2 k+2+r\right. \\
& =\sum_{\substack{\left(I_{1}, I_{2}, I_{3}, I_{4}\right)=I \\
I_{2} \neq \varnothing}} m\left(I_{1}: v_{12 k+2+r}^{-1}\right) m\left(I_{2}: x_{1}\right) m\left(I_{3}: v_{12 k+2+r}\right)^{-1} m\left(I_{4}: x_{1}^{-1}\right) \\
& \equiv m(2, \underbrace{1, \ldots, 1}_{q 1 \text { l's }}: v_{12 k+2+r}) \quad\left(\bmod \Lambda\left(J_{k}\right)\right) \quad(\text { by induction }) .
\end{aligned}
$$

Proof of 4.12.2.

$$
\begin{aligned}
& L\left(I_{k}\right)=\Delta(2, \underbrace{1, \ldots, 1}_{2 k+1 \text { l's }}) \\
& =\operatorname{gcd}\left\{m(2, \underbrace{1, \ldots, 1}_{l \text { l's }}: v_{1}{ }_{2 k+2+r}) \mid 0 \leq l \leq 2 k-1\right\} \quad(\text { Lemma 3.3) } \\
& \equiv 0 \quad\left(\bmod \Lambda\left(J_{k}\right)\right) \quad(\text { Lemma 4.13) } \\
& \sigma\left(J_{k}\right)\left(\omega_{1}\right) \equiv m(2, \underbrace{1, \ldots, 1}_{2 k-1 \text { 1's }}, 2,1: v_{12 k+2+r} x_{1} v_{12 k+2+r}^{-1} x_{1}^{-1}) \\
& \equiv m(2, \underbrace{1, \ldots, 1}_{2 k-1 \text { 1's }}, 2: v_{12 k+2+r}) \quad\left(\bmod \Lambda\left(J_{k}\right)\right) \quad(\text { Lemmas 3.4.2 and 4.13) } \\
& \equiv-T\left(I_{k}\right)(\omega) \text {. }
\end{aligned}
$$

Using results of [1], one can construct links such that $\Lambda\left(J_{k}\right)$ is a proper divisor of $L\left(I_{k}\right)$, or if the linking number of the components is not zero, $L\left(I_{k}\right)$ is a proper divisor of $L_{V}\left(J_{k}\right)$. For $1 \leq l \leq 2 k-2$,

$$
m(2, \underbrace{1, \ldots, 1}_{l 1 \text { 's }}, 2,1: v_{12 k+2+r} x_{1} v_{12 k+2+r}^{-1} x_{1}^{-1})
$$

enters into $\Lambda\left(J_{k}\right)$, but the corresponding term,

$$
m(2, \underbrace{1, \ldots, 1}_{l \text { l's }}, 2: v_{12 k+2+r}),
$$

does not contribute to $L\left(I_{k}\right)$. Furthermore,

$$
m(2, \underbrace{1, \ldots, 1}_{2 k-1 \text { l's }}: v_{12 k+2+r})
$$

and

$$
m(\underbrace{1, \ldots, 1}_{2 k-1 \text { l's }}, 2: v_{12 k+2+r})
$$

contribute to $L\left(I_{k}\right)$, but the corresponding terms,

$$
m(2, \underbrace{1, \ldots, 1}_{2 k \text { l's }}: v_{12 k+2+r} x_{1} v_{12 k+2+r}^{-1} x_{1}^{-1})
$$

and

$$
m(\underbrace{1, \ldots, 1}_{2 k 1 \text { 's }}, 2: v_{12 k+2+r} x_{1} v_{12 k+2+r}^{-1} x_{1}^{-1}),
$$

do not contribute to $L_{V}\left(J_{k}\right)$. 


\section{REFERENCES}

1. T. D. Cochran, Geometric invariants of link cobordism, Comment. Math. Helv. 60 (1985), 291-311.

2. __ Derivatives of links: Milnor's concordance invariants and Massey's products, preprint.

3. W. G. Dwyer, Homology, Massey products, and maps between groups, J. Pure and Appl. Algebra 6 (1975), 177-190.

4. R. Fenn, Techniques of geometric topology, London Math. Soc. Stud. Text, No. 57, Cambridge Univ. Press, Cambridge, 1983.

5. D. Kraines, Massey higher products, Trans. Amer. Math. Soc. 124 (1966), 431-449.

6. W. S. Massey, Higher order linking numbers, Conf. on Algebraic Topology (Univ. of Illinois at Chicago Circle, 1968), Univ. of Chicago at Chicago Circle, Chicago, Ill., pp. 174-205.

7. __ Some higher order cohomology operations, Symposium International de Topologia Algebraica, 1958, pp. 145-154.

8. J. Milnor, Link groups, Ann. of Math. (2) 2 (1954), 177-195.

9. __ Isotopy of links, Algebraic Geometry and Topology: A Symposium in Honor of Solomon Lefshetz, Princeton Univ. Press, Princeton, N.J., 1957, pp. 280-306.

10. R. Porter, Milnor's $\bar{\mu}$-invariants and Massey products, Trans. Amer. Math. Soc. 257 (1980), 39-71.

11. D. Rolfsen, Knots and links, Publish or Perish, Berkeley, California, 1976.

12. N. A. Sato, Cobordism of semi-boundary links, Topology Appl. 18 (1984).

13. J. Stallings, Homology and central series of groups, J. Algebra 2 (1965), 170-181.

14. D. W. Stein, Massey products in the cohomology of groups with applications to link theory, $\mathrm{Ph} . \mathrm{D}$. Thesis, Brandeis Univ., 1986.

15. __ Computing Massey product invariants of links, Proc. 1987 Georgia Topology Conference, Topology Appl. (to appear).

16. V. G. Turaev, The Milnor invariants and Massey products, Studies in Topology. II, Zap. Nauchn. Sem. Leningrad. Otdel. Mat. Kogo oi Stet. Acad. Nauk USSR 66 (1976) (translated in J. Soviet Math.).

Department of Mathematics, Texas A\& M University, College Station, Texas 77843

Current address: Naval Ocean Systems Center, Code 632 (B), San Diego, California 92152-5000 